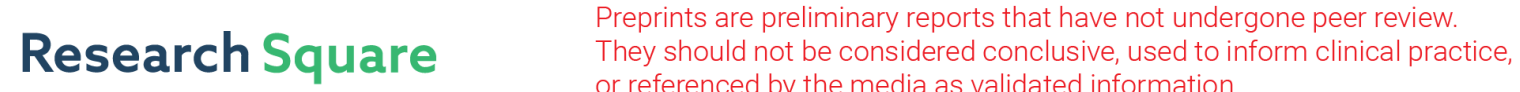 or referenced by the media as validated information. \\ Complex Dynamical Behaviors in a Bertrand Game with Service Factor and Differentiated Products
}

Wei Zhou ( $\nabla$ wei_zhou@vip.126.com )

Lanzhou Jiaotong University https://orcid.org/0000-0002-4477-3940

Hui Li

Lanzhou Jiaotong University

\section{Research Article}

Keywords: Service Spillover, Product Differentiation, Saddle-homoclinic Bifurcation, Intermittent Chaos, Global Dynamics, Critical Curves

Posted Date: April 19th, 2021

DOl: https://doi.org/10.21203/rs.3.rs-397812/v1

License: (1) This work is licensed under a Creative Commons Attribution 4.0 International License.

Read Full License

Version of Record: A version of this preprint was published at Nonlinear Dynamics on September 21st, 2021. See the published version at https://doi.org/10.1007/s11071-021-06897-z. 


\title{
Complex Dynamical Behaviors in a Bertrand Game with Service Factor and Differentiated Products
}

\author{
Wei ZHOU a,b,* Hui LI ${ }^{\mathrm{a}, \mathrm{b}}$ \\ a. School of Mathematics and Physics, Lanzhou Jiaotong University, Lanzhou Gansu 730070, China \\ b. Research Centre of Game theory and Economics Mathematics, Lanzhou Jiaotong University, Lanzhou Gansu 730070, China
}

\begin{abstract}
In this paper, taking the factor of service level provided by the manufacturers into consideration, a static duopolistic Bertrand game with service factor is studied first, in which these two oligarchs produce differentiated products. A dynamic game model of duopoly Bertrand with boundedly rational is established with using the gradient mechanism. By using numerical simulation tools, there are two paths for the system to drop into chaos, that is, flip bifurcation and Neimark-Sacker bifurcation. The symmetric structures can be found from two-parameter bifurcation diagrams. Saddle-homoclinic bifurcation also can be observed from the evolution process of phase portraits. In addition, the emergence of intermittent chaos implies that the established system has the capability of self-regulating, where PM-I intermittency, PM-III intermittency and crisis-induced intermittency have been studied. With the help of the critical curves, the qualitative changes on the basin of attraction are investigated. At last, it can be found that the values of product differentiation degree and service spillover effect are not the bigger the better. Keeping these two parameters in a relatively small range will be conducive to the long-term stable operation of the two manufacturers.
\end{abstract}

Keywords: Service Spillover; Product Differentiation; Saddle-homoclinic Bifurcation; Intermittent Chaos; Global Dynamics; Critical Curves

\section{Introduction}

When the consumers enter the shopping malls to buy goods, they will feel an increasingly common phenomenon, that is, the difference in the function and utility of the same kind of products on the shelf is getting smaller and smaller. The products themselves tend to be homogeneous. One of main reason for the gradual homogenization between the products is the rapid improvement of enterprise production technology. A traditional view on the degree of product differentiation proposed by Shy [1], that is, the reduction of product differentiation may lead to the increase of market competition intensity and lead to price war among enterprises. Although the price competition is the key method for oligopoly market competition to enlarge market shares, no one can deny that conducting a long-term price war will lead to vicious price competition among enterprises, which will further damage the profits of enterprises [2]. This low-level price competition is not conductive to stable operation in long-run. Caplin \& Nalebuff [3] investigated a price competition model with differentiated products and proposed a new method to analyze this imperfect competition mode. Liu et al. [4] studied a mixed duopoly game with endogenous product differentiation and found that the difference between products arises only when the production cost is very low. The differentiation between products can attract the consumers who love a certain feature of the product, and make the consumers distinguish it from

*Email of Corresponding author: wei_zhou@vip.126.com 
the enterprises selling the same kind of products, so that the enterprises producing differentiated products can gain competitive advantages in market competition.

In many literatures on oligopoly market competition, a large number of scholars focus on the degree of product differentiation. Zhang et al. [5] exploited the form of linear inverse demand function [6] including the product differentiation parameter, and then established a dynamic duopoly model with differentiated products and limited rationality. He also investigated the dynamical characteristics of this dynamic model. On the basis of Ref. [5], Ahmed et al. [7] and Anufriev et al. [8] further studied the price competition game between oligopoly that produce differentiated products. Zhang et al. [9] studied complex dynamics in a quantum Bertrand duopoly with differentiated products. It was found that the heterogeneity of products does affect the stability of the game model. In addition, Fanti \& Gori [10] investigated a Cournot game with two heterogeneous oligarchs and analyzed the influence of changes in consumer demand for differentiated products on the stability of the model. He observed that the increase in the degree of differentiation between products can reduce the intensity of competition. Same conclusion could be found in the research of Agliari et al. [11], that is, the higher the differentiation level between products, the smaller the intensity of market competition.

In the process of consumption, consumers pay more and more attention to some non-price factors of products [12], especially the factor like product service level. An increasing number of enterprises and manufacturers begin to attach importance to the aspect of product service. For instance, the clothing manufacturers will present small items such as buttons when selling clothes, online shopping on the e-commerce platform can be delivered to your home directly(amazon.com can deliver free of charge when the consumption limit is reached), and electronic product manufacturers such as Apple and Huawei set up product experience stores. Both enterprises and manufacturers expect to win the favor of consumers by providing product services, and then they can obtain a great deal of market shares. A lot of researchers have made many related researches on this topic. Giri \& Sarker [12] considered a situation that two independent retailers compete in both price level and service level, and analyzed the equilibrium behaviors of a Stackelberg model. Shin [13] pointed that the retailer who provide product service has the advantage of after-sale service. Wu [14] built a competitive game model for sellers to provide information services for horizontal differentiated products. The study found that the motivation to provide information services not only affected the profits of sellers, but also affected social welfare. However, a feature of providing product service that cannot be ignored is service intangibility. After providing product services, it is inevitable that consumers will experience product services from one manufacturer and then purchase products from other manufacturers. This is the notable phenomenon of "free riding" between manufacturers proposed by Telser [15] as early as the early 1960s. In Ref. [13], the scholar Shin put forward that the phenomenon of "free riding" can make free riders reduce the motivation of price competition with service providers. Bernstein [16] also believed that "free riding" among manufacturers is widespread and common, but it does not necessarily have a negative impact on competition. The scholars Tu et al. [17] incorporated service factors into a dynamic hybrid supply chain model, and studied the impacts of the price adjustment speed and the service level on the model. Furthermore, in the Ref. [18-21], the factors of service level or service spillover effect were considered in the establishment of supply chain model. Unfortunately, few literatures use the knowledge of nonlinear dynamics and numerical simulation tools to study these dynamic game models. 
Many singular and interesting dynamic behaviors have appeared in the nonlinear dynamic game models. Fanti et al. [22-24] established the nonlinear duopoly game model with producing differentiated products and carrying on management entrustment agent, and chose the degree of product differentiation as important parameter to investigate. It could be found that the qualitative structures of attracting basin will have great changes after global bifurcation, the fractal structures and the coexisting phenomenon between attractors will be generated. Bischi et al. [25-28] also found the phenomenon of coexistence between the attractors in the basin of attraction, when he studied the economic dynamic game models. Bischi assumed that the global bifurcation occurs in the system may due to the critical curves contact with the borders of attracting basin, which cause the dramatic changes in size, structure and shape of basin of attraction. Zhang et al. [29] studied the complex dynamic behaviors appearing in a semi-collusion Cournot duopoly game model, in which the enterprises cooperate in production but compete in process of research and development. Zhou et al. [30] observed intermittency phenomenon in the built two-stage dynamic game, in which both two enterprises carried on research and development activities. Zhou et al. [31] not only found the phenomenon of coexisting attractors in the attracting basin, but also used critical curves to analyze the reason for the structural change of the basin of attraction. A lot of relative investigations can be found in Refs. [32-34].

The rest parts of this research are as follows: a static duopolistic Bertrand game is studied in Sec.2. In Sec.3, a duopoly Bertrand dynamic game model with the gradient mechanism to adjust pricing strategy is established. The analyses on local stability of the two-dimensional discrete noninvertible dynamic system are given in details. With the help of numerical simulation tools, the paths to chaos and the symmetric structures in $\left(b_{1}, b_{2}\right)$-plane are analyzed in Sec.4. In Sec.5, the intermittency phenomena are discussed. The multi-stability in the basin of attraction is shown in Sec.6. In Sec.7, the analyses on profits bifurcation are given. At last, some valuable conclusions are primarily given in Sec.8.

\section{Static duopolistic Bertrand game with service factor}

With the continuous improvement of production technology, the products produced by different manufacturers tend to be homogeneity-oriented. That is to say, the differences in quality, function and utility between products are very small. On the basis that there is no restriction on the production capacity of manufactures (namely, the manufactures dose not compete in outputs), if one manufacturer attempts to cut the price of its own products so as to increase sales, then other manufacturers will follow the price reduction measures one after another, which will lead the vicious price competition among manufacturers [1]. The final result of vicious price war can only be that the selling price of products will drop again and again until it is equal to the marginal cost of production and then the manufacturers cannot make a profit anymore. On the contrary, the case that one manufacturer will occupy a large market share due to its price reduction measures will not arise, if there are differences between the products produced by different manufacturers. This is because that there will always be loyal consumers who have a preference for a certain feature of the product and will not be shaken by price changes [10]. Hence, the manufacturers should consider increasing product differentiation to ease the dilemma caused by vicious price competition [11]. Furthermore, during the process of consumption, more and more consumers pay attention on non-price factors such as product service. The product service provided by 
manufacturers is an effective way to avoid price wars, compete for consumer preferences and gain large market share, and then enhance the competitive advantage of manufacturers in the market. This service measure also can turn manufacturers from a single price competition mode to a multi-dimensional competitive mode including price and service [12].

It is assumed that there are two oligarchs existing in an industry, labeled by $M_{i}(i=1,2)$, and these two manufacturers produce differentiated products and provide product services. The selling price $p_{i}(t)$ and the product outputs $q_{i}(t)$ of the manufacturer $M_{i}$ during $t$ period satisfy the following form of linear demand function:

$$
q_{i}(t)=a-b_{i} p_{i}(t)+\gamma_{i} p_{j}(t)+\oiint_{t}, \quad i, j=1,2, \quad i \neq j, \quad t \in ¥,
$$

where $a>0$ denotes the largest scale of the market, $b_{i}>0$ represents price sensitivity coefficient, and $\gamma_{i}$ represents the product differentiation coefficient. Moreover, the parameter $\gamma_{i}$ belongs to $[0,1)$ and $0<\gamma_{i}<b_{i}$. The smaller the value of $\gamma_{i}$, the greater the degree of differentiation between products and $\gamma_{i}=0$ denotes that the products produced by manufacturers $M_{i}$ are totally heterogeneous. Since providing product services have the character of service intangibility, it is impossible for manufacturers to avoid the problem that consumers experience product service in their own retail stores but buy products from their competitors. That is to say, there will be spillover effect of services, which will lead to the "free riding" phenomenon [15]. It is further assumed that the service level that the consumers experience from the manufacturer $M_{i}$ is marked as $\oiint_{i}$ in Eq.(1). It satisfies the equation that $\oiint_{l}=s_{i}+\theta_{j} s_{j}(i, j=1,2, i \neq j)$, where $s_{i}>0$ and $s_{j}>0$ respectively represent the service level provided by two oligarchs. The parameter $\theta_{j} \in(0,1]$ denotes the intensity of service spillover effect. Then the cost functions of these two oligarchic manufacturers $M_{i}$ have linear forms, which can be expressed as follows,

$$
C_{i}\left(q_{i}\right)=c_{i} q_{i}(t)
$$

in which $c_{i}>0$ is the marginal production cost that producing unit product. The revenue functions with service spillover effect of two duopoly manufacturers in $t$ period may be expressed as following,

$$
\pi_{i}(t)=\left(p_{i}(t)-c_{i}\right) q_{i}(t)-S_{i},
$$


where $S_{i}=\frac{1}{2} h s_{i}^{2}$ represents the service cost that a manufacturer $M_{i}$ needs to spend when providing a service level as $s_{i}$, and $h>0$ is the service cost coefficient. In this research, we mainly discuss and analyze the case that $b_{1}=b_{2}=b, \gamma_{1}=\gamma_{2}=\gamma$ and $\theta_{1}=\theta_{2}=\theta$. Based on above assumptions, a static duopolistic game has been established, in which the manufacturers compete in the price $p_{i}$ of the products and both of them pursue profit maximization. Under this duopolistic setting, the following theorem is tenable.

Proposition 1. The unique Nash equilibrium of the static Bertrand game exists and it can be expressed as

$$
P_{N}^{*}=\left(\frac{2 b\left(a+s_{1}+\theta s_{2}+b c_{1}\right)+\gamma\left(a+s_{2}+\theta s_{1}+b c_{2}\right)}{4 b^{2}-\gamma^{2}}, \frac{2 b\left(a+s_{2}+\theta s_{1}+b c_{2}\right)+\gamma\left(a+s_{1}+\theta s_{2}+b c_{1}\right)}{4 b^{2}-\gamma^{2}}\right) .
$$

Proof. Both two manufacturers want to maximize their respective profits, which means that the first order conditions of their revenue functions need to be equal to zero. That is, the marginal revenues are equal to zero. In addition, the corresponding marginal revenue function of manufacturer $M_{i}$ can be given as

$$
\frac{\partial \pi_{i}(t)}{\partial p_{i}(t)}=a-2 b p_{i}+\gamma p_{j}+s_{i}+\theta s_{j}+b c_{i} .
$$

Then, the Nash equilibrium can be obtained by calculating the algebraic system as following:

$$
\left\{\begin{array}{l}
\frac{\partial \pi_{1}(t)}{\partial p_{1}(t)}=a-2 b p_{1}+\gamma p_{2}+s_{1}+\theta s_{2}+b c_{1}=0 \\
\frac{\partial \pi_{2}(t)}{\partial p_{2}(t)}=a-2 b p_{2}+\gamma p_{1}+s_{2}+\theta s_{1}+b c_{2}=0
\end{array} .\right.
$$

From the first equation, we can easily get a relation that $p_{1}=\frac{a+\gamma p_{2}+s_{1}+\theta s_{2}+b c_{1}}{2 b}$. By substituting this relation into the second equation of the algebraic system, it can be obtained that $p_{2}^{*}=\frac{2 b\left(a+s_{2}+\theta s_{1}+b c_{2}\right)+\gamma\left(a+s_{1}+\theta s_{2}+b c_{1}\right)}{4 b^{2}-\gamma^{2}}$. Finally, the value of $p_{1}^{*}$ can be gained by retrieving the value of $p_{2}^{*}$, namely, $p_{1}^{*}=\frac{2 b\left(a+s_{1}+\theta s_{2}+b c_{1}\right)+\gamma\left(a+s_{2}+\theta s_{1}+b c_{2}\right)}{4 b^{2}-\gamma^{2}}$. Therefore, this static duopolistic Bertrand game has only one Nash equilibrium $P_{N}^{*}=\left(p_{1}^{*}, p_{2}^{*}\right)$.

\section{Dynamic game with the gradient mechanism}

In real market, gathering market information is costly, so it is difficult for firms to grasp all the market information and make competitive decisions completely rationally. In order to accord with actual market situation, we further suppose that these two manufacturers considered in this model are bounded rational and will adopt the gradient mechanism [35-36]. That is to say, each 
manufacturer will adjust the selling price of the products in $t+1$ period by estimating its marginal profit in $t$ period. Moreover, both these two manufacturers make long-term product pricing decisions through repeated competitive games. The selling price of products in $t+1$ period is

$$
p_{i}(t+1)=p_{i}(t)+k_{i} p_{i}(t) \frac{\partial \pi_{i}(t)}{\partial p_{i}(t)}
$$

where $k_{i}>0$ denotes the adjustment speed of pricing decision of the manufacturer $M_{i}$, which also means that the difference between the price in $t+1$ period and the price in $t$ period is directly proportional to its marginal profit in $t$ period. Finally, the price dynamic adjustment model of two boundedly rational oligarchs who produce differentiated products and provide services could be expressed as following,

$$
\left\{\begin{array}{l}
p_{1}(t+1)=p_{1}(t)+k_{1} p_{1}(t)\left[a-2 b p_{1}(t)+\gamma p_{2}(t)+s_{1}+\theta s_{2}+b c_{1}\right] \\
p_{2}(t+1)=p_{2}(t)+k_{2} p_{2}(t)\left[a-2 b p_{2}(t)+\gamma p_{1}(t)+s_{2}+\theta s_{1}+b c_{2}\right]
\end{array} .\right.
$$

To further facilitate calculation and analysis of the system (7), we introduce auxiliary variables that $A=a+s_{1}+\theta s_{2}+b c_{1}$ and $B=a+s_{2}+\theta s_{1}+b c_{2}$. Then the system (7) can be simplified as

$$
T:\left\{\begin{array}{l}
p_{1}(t+1)=p_{1}(t)+k_{1} p_{1}(t)\left[A-2 b p_{1}(t)+\gamma p_{2}(t)\right] \\
p_{2}(t+1)=p_{2}(t)+k_{2} p_{2}(t)\left[B-2 b p_{2}(t)+\gamma p_{1}(t)\right]
\end{array},\right.
$$

in which $T$ is time evolution operator.

\subsection{Local stability analysis}

It is assumed that these two manufacturers are naïve. Then they think the price of their opponent in current period is equal to the selling price in previous period, that is, $p_{1}(t+1)=p_{1}(t)$ and $p_{2}(t+1)=p_{2}(t)$. The marginal revenue of two manufacturers will equal to zero and the system $T$ will achieve local equilibrium, then we can get two equations as following,

$$
\left\{\begin{array}{l}
A-2 b p_{1}+\gamma p_{2}=0 \\
B-2 b p_{2}+\gamma p_{1}=0
\end{array}\right. \text {. }
$$

After some simple calculation steps, it is not hard to find out that the system $T$ includes four different equilibrium points, that is, $P_{1}=(0,0), \quad P_{2}=\left(0, \frac{B}{2 b}\right), \quad P_{3}=\left(\frac{A}{2 b}, 0\right) \quad$ and $P_{N}^{*}=\left(\frac{2 A b+B \gamma}{4 b^{2}-\gamma^{2}}, \frac{2 B b+A \gamma}{4 b^{2}-\gamma^{2}}\right)$. In addition, the points $P_{1}, P_{2}$ and $P_{3}$ are boundary equilibrium points.

We are going to analyze the local stability of these four equilibria next. The corresponding Jacobian matrix of the system $T$ will be given as following,

$$
J(T)=\left(\begin{array}{ll}
J_{11} & J_{12} \\
J_{21} & J_{22}
\end{array}\right)
$$


where $\quad J_{11}=\frac{\partial p_{1}(t+1)}{\partial p_{1}(t)}=1+k_{1}\left(A-4 b p_{1}+\gamma p_{2}\right), \quad J_{12}=\frac{\partial p_{1}(t+1)}{\partial p_{2}(t)}=k_{1} \gamma p_{1}, \quad J_{21}=\frac{\partial p_{2}(t+1)}{\partial p_{1}(t)}=k_{2} \gamma p_{2}$ and $J_{22}=\frac{\partial p_{2}(t+1)}{\partial p_{2}(t)}=1+k_{2}\left(B-4 b p_{2}+\gamma p_{1}\right)$. From the non-negativity of the above parameters, $A>0$ and $B>0$, the following theorems hold.

Proposition 2. The boundary equilibrium point $P_{1}=(0,0)$ is an unstable node.

Proof. See Appendix A.

Proposition 3. When the adjustment speed $k_{2}$ of the manufacturer $M_{2}$ satisfies the condition that $k_{2}<\frac{2}{B}$, then the boundary equilibrium point $P_{2}=\left(0, \frac{B}{2 b}\right)$ is a saddle point. Otherwise, $P_{2}=\left(0, \frac{B}{2 b}\right)$ is an unstable node.

Proof. See Appendix A.

The corresponding Jacobian matrix $J\left(P_{N}^{*}\right)$ of the system $T$ at Nash equilibrium $P_{N}^{*}=\left(\frac{2 A b+B \gamma}{4 b^{2}-\gamma^{2}}, \frac{2 B b+A \gamma}{4 b^{2}-\gamma^{2}}\right)$ can be written as

$$
J\left(P_{N}^{*}\right)=\left(\begin{array}{cc}
1+\frac{2 k_{1} b \gamma B+4 k_{1} b^{2} A}{\gamma^{2}-4 b^{2}} & \frac{2 b k_{1} \gamma A+k_{1} \gamma^{2} B}{4 b^{2}-\gamma^{2}} \\
\frac{2 b k_{2} \gamma B+k_{2} \gamma^{2} A}{4 b^{2}-\gamma^{2}} & 1+\frac{2 k_{2} b \gamma A+4 k_{2} b^{2} B}{\gamma^{2}-4 b^{2}}
\end{array}\right) .
$$

The characteristic equation of $J\left(P_{N}^{*}\right)$ is given as following

$$
E(\lambda)=\lambda^{2}-\operatorname{Tr}\left(P_{N}^{*}\right) \lambda+\operatorname{Det}\left(P_{N}^{*}\right)
$$

in which $\operatorname{Tr}\left(P_{N}^{*}\right)$ and $\operatorname{Det}\left(P_{N}^{*}\right)$ are the trace and the determinant of the matrix $J\left(P_{N}^{*}\right)$, respectively. The specific expressions of $\operatorname{Tr}\left(P_{N}^{*}\right)$ and $\operatorname{Det}\left(P_{N}^{*}\right)$ are

$$
\begin{gathered}
\operatorname{Tr}\left(P_{N}^{*}\right)=2+\frac{A\left(2 k_{2} b \gamma+4 k_{1} b^{2}\right)+B\left(2 k_{1} b \gamma+4 k_{2} b^{2}\right)}{\gamma^{2}-4 b^{2}}, \\
\operatorname{Det}\left(P_{N}^{*}\right)=1+\frac{\gamma^{2} k_{1} k_{2} A B-2 b \gamma\left(k_{2} A+k_{1} B\right)+2 \gamma k_{1} k_{2} b\left(B^{2}+A^{2}\right)-4 b^{2}\left(k_{1} A+k_{2} B\right)+4 b^{2} k_{1} k_{2} B A}{4 b^{2}-\gamma^{2}} .
\end{gathered}
$$

Proposition 4. The system $T$ will never lose its stability at Nash equilibrium $P_{N}^{*}$ through Neimark-Sacker bifurcation.

Proof. The discriminant of the characteristic equation $E(\lambda)$ can be given as

$$
\Delta=\operatorname{Tr}^{2}\left(P_{N}^{*}\right)-4 \operatorname{Det}\left(P_{N}^{*}\right) .
$$

Since the non-negativity of the parameters and $4 b^{2}-\gamma^{2}>0$, it is quite obvious that the 
discriminant $\Delta$ is always greater than zero. This means that the characteristic equation $E(\lambda)$ has two different real eigenvalues. Namely, the system will never lose its stability through Neimark-Sacker bifurcation.

Proposition 5. When the adjustment speeds $k_{1}$ and $k_{2}$ respectively satisfy the conditions that $k_{1}>\frac{4 b}{\gamma B}$ and $k_{2}>\max \left\{\frac{4 b}{\gamma A}, \frac{\gamma}{b A}\right\}$, the unique Nash equilibrium $P_{N}^{*}$ is locally stable. Otherwise, the system $T$ will enter into chaos via flip bifurcation.

Proof. The sufficient conditions for local stability of the unique point $P_{N}^{*}=\left(\frac{2 A b+B \gamma}{4 b^{2}-\gamma^{2}}, \frac{2 B b+A \gamma}{4 b^{2}-\gamma^{2}}\right)$ can be given with the help of Jury criterion, namely, the point $P_{N}^{*}$ is local stable if three stability conditions of Jury criterion hold at the same time. The three conditions of Jury criterion corresponding to Nash equilibrium $P_{N}^{*}$ can be specifically expressed as follows,

(i) $1-T r+\operatorname{Det}=\frac{k_{1} k_{2}\left(\gamma^{2} B A+2 \gamma B^{2} b+2 \gamma A^{2} b+4 b^{2} B A\right)}{4 b^{2}-\gamma^{2}}>0$.

(ii) $1+\operatorname{Tr}+\operatorname{Det}=4+\frac{\left(2 k_{2} b B+k_{2} \gamma A\right)\left(\gamma k_{1} B-4 b\right)+2 k_{1} b A\left(\gamma k_{2} A-4 b\right)+4 k_{1} b B\left(b A k_{2}-\gamma\right)}{4 b^{2}-\gamma^{2}}>0$.

(iii) $1-D e t=\frac{2 b \gamma\left[k_{1} B\left(1-k_{2} B\right)+k_{2} A\left(1-k_{1} A\right)\right]+4 b^{2} k_{2} B\left(1-A k_{1}\right)+A k_{1}\left(4 b^{2}-\gamma^{2} B k_{2}\right)}{4 b^{2}-\gamma^{2}}>0$.

We can know from the non-negativity of the parameters and the existence of Nash equilibrium $P_{N}^{*}$ that the condition (i) always holds, so there is no transcritical bifurcation, or saddle-node bifurcation, or pitchfork bifurcation will take place. From Theorem 4, we can also know that the condition (iii) is satisfied. In addition, when the adjustment speeds $k_{1}$ and $k_{2}$ of two manufacturers $M_{1}$ and $M_{2}$ respectively satisfy the conditions that

$$
\begin{gathered}
k_{1}>\frac{4 b}{\gamma B}, \\
k_{2}>\max \left\{\frac{4 b}{\gamma A}, \frac{\gamma}{b A}\right\},
\end{gathered}
$$

the condition (ii) holds. The unique Nash equilibrium point $P_{N}^{*}=\left(\frac{2 A b+B \gamma}{4 b^{2}-\gamma^{2}}, \frac{2 B b+A \gamma}{4 b^{2}-\gamma^{2}}\right)$ of the system $T$ is locally stable. Otherwise, the system $T$ may experience a flip bifurcation when $1+T r+D e t=0$, which will cause the system $T$ to become unstable and gradually deviate from Nash equilibrium point $P_{N}^{*}=\left(\frac{2 A b+B \gamma}{4 b^{2}-\gamma^{2}}, \frac{2 B b+A \gamma}{4 b^{2}-\gamma^{2}}\right)$, even fall into chaos.

\section{Numerical simulation and illustration}

In the previous section, the local stabilities for the four equilibrium points are given by taking use 
of the knowledge of nonlinear dynamics and the Jury criterion. It can be known that the system $T$ will only lose its stability at the Nash equilibrium through flip bifurcation. Then, in the following sections, we will use tools of numerical simulation to further investigate and discuss the more complex dynamic behaviors that may appear in the system $T$. The main tools of numerical simulation contain bifurcation diagrams, the largest Lyapunov exponents, phase portraits, time-series diagrams and basins of attraction, etc. Apparently, the changes of value of each parameter will have significant impacts on the stability of the system $T$.

\subsection{The path to chaos}

Firstly, the parameters that the speed of adjustment $k_{1}$, the service level $s_{1}$, the service spillover effect $\theta$ and the price sensitivity coefficient $b$ are respectively selected as bifurcation parameters. The other parameters are fixed as $a=4.6198, \gamma=0.3668, k_{2}=0.1496$, $s_{2}=4.0000, c_{1}=1.0524$ and $c_{2}=1.0079$. We are interested in studying the effects of these bifurcation parameters on the stability of the system $T$, especially the influences on the manufacturer 2. The results of numerical simulation under this set of parameter conditions are shown in Fig.1, the bifurcation curves with respect to the price $p_{2}$ of the products produced by $M_{2}$ are denoted as blue and the corresponding largest Lyapunov exponents are marked as black curve. The two 1-D bifurcation diagrams with respect to the speed of adjustment $k_{1}$ are shown together in Fig.1(a). The upper 1-D bifurcation diagram in Fig.1(a), which shows the bifurcation curve that the system $T$ enters into chaos via the path of Neimark-Sacker bifurcation. It is quite remarkable that the values of black curves marked by pink box fluctuate up and down near zero, which means that the system is at quasi-periodic state. The lower figure of Fig.1(a) shows the shape of the bifurcation curve corresponding to flip bifurcation path. In detail, the state of system $T$ changes from steady period- 1 motion to period-2 motion when $k_{1}=0.1699$, and the state will change from period- 2 motion to period- 4 motion when $k_{1}=0.1722$. Finally, the system $T$ will drop into chaos when $k_{1}=0.1788$. 
(a)
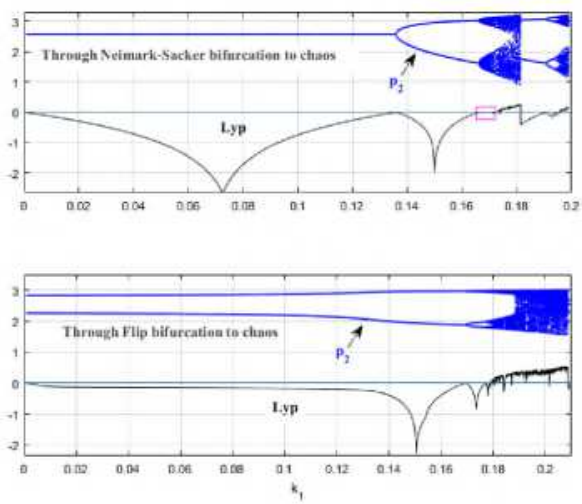

(c)

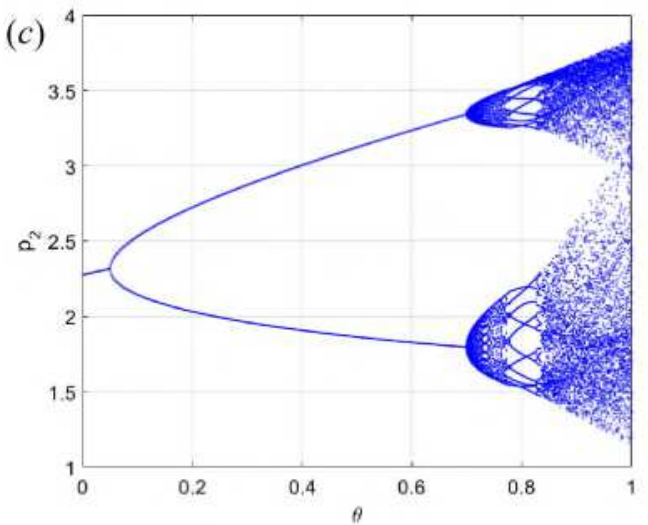

(b)
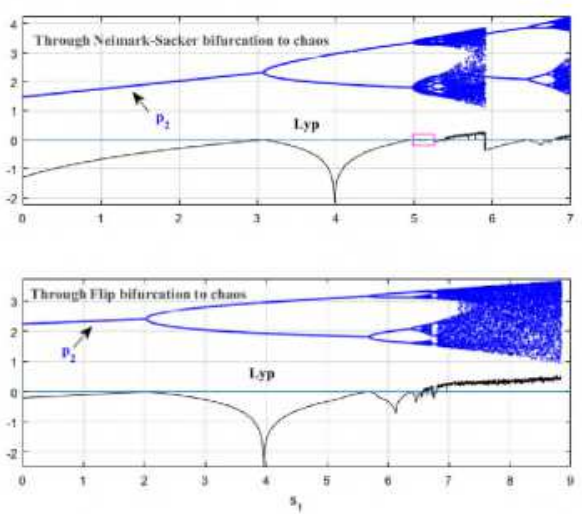

(d)

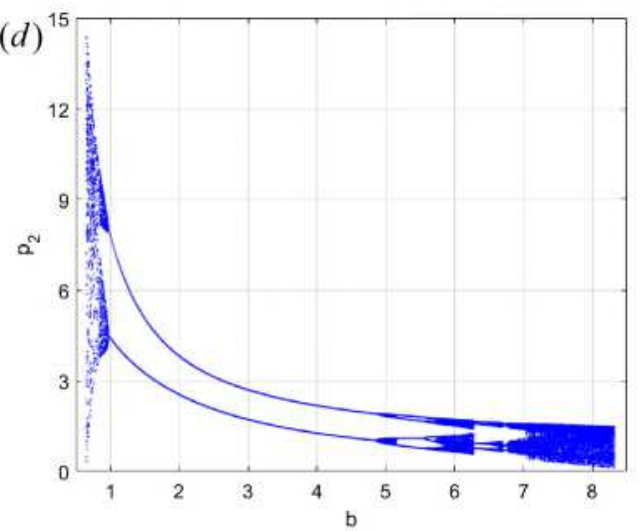

Fig.1. The 1-D bifurcation diagrams with respect to the parameters $k_{1}, s_{1}, \theta$ and $b$. The bifurcation curves of the price $p_{2}$ are denoted as blue and the corresponding largest Lyapunov exponents are marked as black curves. The other parameters are chosen as $a=4.6198, \gamma=0.3668, s_{2}=4.0000, k_{2}=0.1496, c_{1}=1.0524$ and $c_{2}=1.0079$. (a) $b=2.6707, \theta=0.3639, s_{1}=4.0000$. (b) $b=2.6707, k_{1}=0.1514, \theta=0.3639$. (c) $b=2.6707, k_{1}=0.1514, s_{1}=4.0000$. (d) $k_{1}=0.1514, \theta=0.3639, s_{1}=4.0000$.

The two different bifurcation paths with respect to the bifurcation parameter $s_{1}$ are shown together in Fig.1(b). The way of bifurcation shown in the upper figure of Fig.1(b) is similar to that of Fig.1(a). In addition, the largest Lyapunov exponents marked by a pink box fluctuate up and down near zero, which means that the system $T$ is at quasi-periodic state. With constant increase of $s_{1}$, the system $T$ will lose its stability via Neimark-Sacker bifurcation. That is to say, the system will first undergo a period of quasi-periodic state and then enter into chaos. The bifurcation curve corresponding to another bifurcation path of the system $T$ is displayed in the lower figure of Fig.1(b), and the system $T$ is at period-1 stable state when $s_{1} \in[0,2.0280)$. Similarly, with the increase of $s_{1}$, the system $T$ will experience a range of period-doubling bifurcations and eventually enter into chaotic motion when $s_{1}=6.8160$.

The 1-D bifurcation diagrams with regard to $\theta$ and $b$ are given in Fig.1(c) and Fig.1(d), respectively. We can clearly see from Fig.1(c) that the system $T$ will keep in stable state only 
when the value of the spillover effect $\theta$ is relatively small. Once the value of $\theta$ exceeds 0.6970, then the system $T$ will become unstable and unpredictable. Furthermore, it is quite worth to notice that the way of bifurcation in Fig.1(d) is differ from the first three figures in Fig.1. The system $T$ is always at chaotic state until $b$ increases to 0.9860 , then the state of the system $T$ will transfer from chaotic to stable period-1 when $b \in[0.9860,4.8700]$. Finally, the system $T$ will drop into chaos again, when the value of $b$ exceeds 4.8700. In addition, from Fig.1(d), a reasonable assumption will be put forward. That is, the bifurcation diagrams with respect to the price sensitivity coefficient $b$ may have symmetric structure, if under appropriate parameter conditions. Hence, the effect of the parameter $b$ on the stability of the system $T$ needs to be further investigated and analyzed.

\subsection{Symmetric structures in $\left(b_{1}, b_{2}\right)$-plane}

In this subsection, we continue to discuss the case that $\gamma_{1}=\gamma_{2}=\gamma$ and $\theta_{1}=\theta_{2}=\theta$, but we further analyze and study a different and special case that $b_{1} \neq b_{2}$. The parameter conditions are fixed as $a=0.3418, \quad \gamma=0.4363, \quad \theta=0.1739, \quad s_{1}=0.0783, \quad s_{2}=0.0783, \quad k_{1}=0.9547$, $k_{2}=0.9547, c_{1}=1.2727$ and $c_{2}=1.2727$. The 2-D bifurcation diagrams with regard to the price sensitivity coefficient $b_{1}$ and $b_{2}$ are shown in Fig.2. The color bar is located on the right and different colors in the color bar represent different periods of the system $T$. In addition, the color black represents the system $T$ may be at quasi-periodic motion or chaotic motion, while the color white represents that if the values of $b_{1}$ and $b_{2}$ are chosen from this area, the trajectories of the system $T$ will be divergent and tend to infinity.

From Fig.2(a), we can clearly see that there are many symmetric structures, Arnold's tongues structures [37] (which are caused by Neimark-Sacker bifurcation) and V-shape islands [38] locate near the period-2 stable area. Then, the other parameter conditions are unchanged and we only change the values of $c_{1}$ and $c_{2}$ to $c_{1}=1.3396$ and $c_{2}=1.3396$. As is shown in Fig.2(b), the period-2 stable area will disappear but the symmetric properties will keep unchanged. As is shown in Fig.2(c), the marginal production costs are given as $c_{1}=1.4022$ and $c_{2}=1.4022$. From Fig.2(c), it is clear that many Arnolds' tongues structures have been disappeared. Compared to the period-14 purple structure in the upper right corner in Fig.2(a) and Fig.2(b), it obviously moves down to the center and is surrounded by light green periodic structures in Fig.2(c). Furthermore, the size of this period-14 purple structure in Fig.2(c) apparently gets bigger and there is a black hole appears in the center of this area. With constant increase of $c_{1}$ and $c_{2}$, the period-14 purple structure will also disappear and the period-18 light pink structure located in the lower left corner will move up, as shown in Fig.2(d). In Fig.2(d), the period-18 light pink structure is entangled by 
the period-20 red band structures and its size apparently gets bigger.

However, with the values of $c_{1}$ and $c_{2}$ continually increase to $c_{1}=1.4754$ and $c_{2}=1.4754$, the light pink structure not exists anymore, only the period-20 red band structures still exist( see Fig.2(e)). Finally, as is shown in Fig.2(f), the marginal production costs are given as $c_{1}=1.5177$ and $c_{2}=1.5177$, respectively. We can clearly find that the symmetric bright pink structures in Fig.2(e) evolve to two symmetric structures with small ring in Fig.2(f). In $\left(b_{1}, b_{2}\right)$-plane, the stable region located on the diagonal, the V-shape structures and Arnold's tongues structures located on both sides will disappear with changing the values of $c_{1}$ and $c_{2}$.
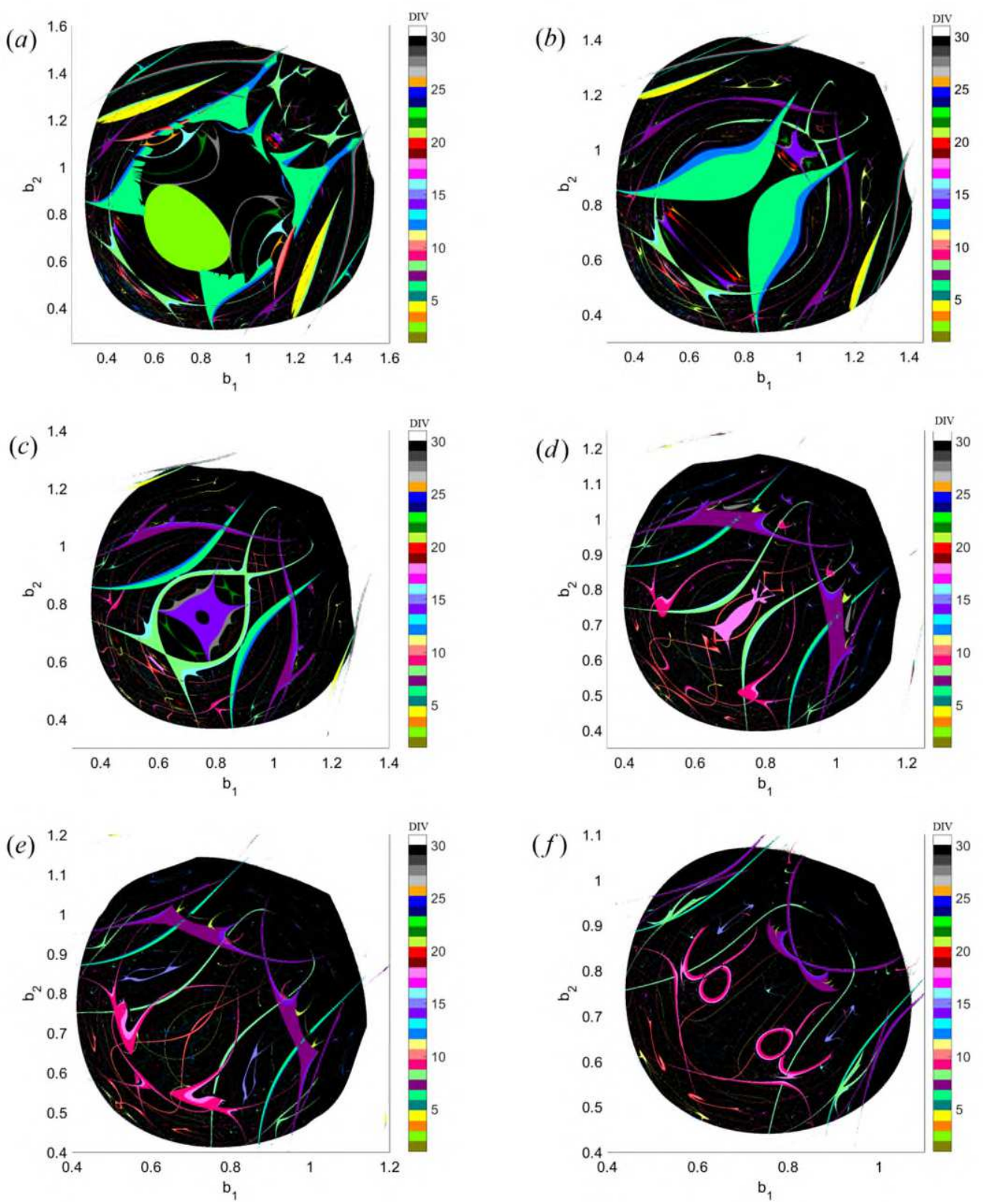

Fig.2. The 2-D bifurcation diagrams in $\left(b_{1}, b_{2}\right)$-plane. The parameter conditions are chosen as $a=0.3418$, 
$\gamma=0.4363, \quad \theta=0.1739, \quad k_{1}=0.9547, \quad k_{2}=0.9547, \quad s_{1}=0.0783, \quad s_{2}=0.0783 \quad$. (a) $c_{1}=1.2727 \quad$ and

$c_{2}=1.2727$. (b) $c_{1}=1.3396$ and $c_{2}=1.3396$. (c) $c_{1}=1.4022$ and $c_{2}=1.4022$. (d) $c_{1}=1.4539$ and

$c_{2}=1.4539$. (e) $c_{1}=1.4754$ and $c_{2}=1.4754$. (f) $c_{1}=1.5117$ and $c_{2}=1.5117$.

\subsection{Saddle-homoclinic bifurcation}

In addition to the two different bifurcation paths of the system $T$ discussed above, we can also find another type of bifurcation existing in the attractor evolution of a set of phase portraits. As is known to all, when the times of iteration tend to be infinity, the phase portrait of the attractor can be regarded as a kind of asymptotic behavior exhibited by the nonlinear dynamic system. Then the phase portraits can characterize final motion of nonlinear dynamic system. Moreover, to better understand the final evolvement is one of the core concerns for this research.

The 1-D bifurcation diagram of product prices $p_{1}$ and $p_{2}$ about the service spillover effect $\theta$ and the corresponding 1-D largest Lyapunov exponent diagram can be seen in Fig.3(a), in which the parameter conditions are fixed as $a=4.9196, b=1.2998, \gamma=0.6907, k_{1}=0.1676$, $k_{2}=0.1675, s_{1}=2.0513, s_{2}=2.4338, c_{1}=0.2287 \quad$ and $c_{2}=1.1081$. In Fig.3(a), the bifurcation curve of the product price $p_{1}$ of $M_{1}$ is marked by red, and that of the price $p_{2}$ of $M_{2}$ is marked by blue. With the value of $\theta$ increasing constantly, the system $T$ will undergo Neimark-Sacker bifurcation when $\theta \approx 0.1737$. The figure pointed by a pink arrow is an enlargement view of the largest Lyapunov exponent diagram corresponding to the pink box. From the enlargement diagram, we can clearly observe that the values of the largest Lyapunov exponents fluctuate up and down near zero, when $\theta \in[0.1737,0.2682]$. It means that the system

$T$ will first show quasi-periodic behaviors after experiencing Neimark-Sacker bifurcation.

With constant increase of the service spillover effect $\theta$, the evolution process for the phase portraits of attractors are displayed in Fig.3(b). First, when the value of $\theta$ is selected as 0.1705, the corresponding attractors at this time are two dodger blue foci with rough selvedge. This period-2 dodger blue focus with rough edge will evolve to two orange invariant cycles with rough edge when the value of $\theta$ increases to 0.2000 , which is due to the system $T$ undergo a Neimark-Sacker bifurcation. Then we can go a step further and continually increase the value of $\theta$, the size of the period-2 red invariant cycle will gradually grow up and the rough edge will died away. Until $\theta=0.2652$, the burrs will disappear completely, and then two black invariant cycles with smooth boundary will emerge. When the value of the parameter $\theta$ increases to 0.4416 , these two black invariant cycles with smooth boundary will burst and then the phenomenon of "phase-locking" will appear and form 20-piece of green attractors. Subsequently, with further increase of $\theta$, two invariant cycles with smooth boundary will appear again, and then evolve to two chaotic attractors marked by sky blue when $\theta=0.5094$. The larger the value of the parameter $\theta$ is, the bigger the size of two chaotic attractors. Finally, when $\theta=0.5967$, these two sky blue chaotic attractors will be twisted together to form one-piece of dark blue homoclinic cycle. This phenomenon may be due to the saddle-homoclinic bifurcation, which we cannot find 
in Fig.3(a). In $\left(p_{1}, p_{2}\right)$-plane, the phase portraits of the attractors with respect to different values of $\theta$ are shown together in Fig.3(b), and the size of the attractor will become larger and larger from inside to outside.

(a)



(b)

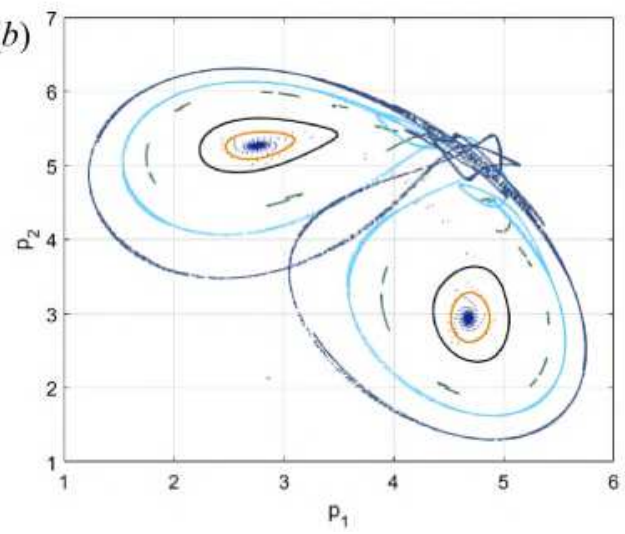

Fig.3. The parameter conditions are chosen as $a=4.9196, b=1.2998, \gamma=0.6907, k_{1}=0.1676, k_{2}=0.1675$, $s_{1}=2.0513, s_{2}=2.4338, c_{1}=0.2287$ and $c_{2}=1.1081$. (a) The 1-D bifurcation diagram with regard to $\theta$ and the corresponding largest Lyapunov exponents, in which red represents the price of $M_{1}$ and blue represents the price of $M_{2}$. The figure pointed by a pink arrow is the enlargement view of the largest Lyapunov exponent diagram marked by the pink box. (b) In $\left(p_{1}, p_{2}\right)$-plane, the evolution process for the phase portraits of attractors with constant increase of $\theta$. From inside to outside, different attractors are respectively marked by dodger blue, orange, black, green, sky blue and dark blue, and the corresponding values of $\theta$ are $\theta=0.1705, \theta=0.2000$, $\theta=0.2652, \theta=0.4416, \theta=0.5094$ and $\theta=0.5967$.

\section{Intermittent chaos}

Among the results of numerical simulation of the system $T$, another notable phenomenon is that the appearance of intermittent chaos can be found in the time series plot, bifurcation diagrams, Lyapunov exponent diagrams, etc. The main characteristic of intermittent chaos in the time series plot is that the amplitude of state variable changes alternately between regular oscillation and irregular oscillation. The motion that the amplitude of state variable changes abruptly and oscillates irregularly is called chaotic motion. Such a phenomenon is so called intermittent chaos. Corresponding to the real economic system, if there is the phenomenon of intermittent chaos occurs in the system, it means that the system has self-regulation ability. That is to say, without the help of external force, the economic system can adjust and reverse the market state of disordered competition to the market state of orderly competition by itself. In addition, back in the early 1980s, two French scholars, Pomeau and Manneville, had subdivided the phenomena of intermittent chaos into three types. Later scholars also named the three kinds of intermittent chaos with the initials of these two scholars as PM-I intermittent chaos, PM-II intermittent chaos and PM-III intermittent chaos [39]. Nowadays, the phenomena of intermittent chaos include five types in general, which are specifically divided into PM-type (PM-I intermittency, PM-II intermittency, PM-III intermittency), On-off and In-out intermittency, and crisis-induced intermittency [40].

The parameter conditions are chosen as $a=2.9147, b=2.8236, \gamma=0.3552, \theta=0.8802$, $k_{2}=0.3744, s_{1}=0.8872, s_{2}=0.2240, c_{1}=0.8811$ and $c_{2}=0.7042$. The bifurcation diagram 
in regard to decision parameter $k_{1}$ is shown in Fig.4(a), and its corresponding 1-D largest Lyapunov exponent diagram is displayed in Fig.4(b). The color red in Fig.4(a) represents the bifurcation curve corresponding to the price $p_{1}$ and blue represents the bifurcation curve corresponding to the price $p_{2}$. When $k_{1}=0.3279$, after undergoing the Neimark-Sacker bifurcation, the system $T$ will directly enter into quasi-periodic motion from period-2 stable motion. Then it will go back to periodic stable state when $k_{1}=0.3356$. Furthermore, the value of the decision adjustment speed $k_{1}$ marked by a light blue line in Fig.4(a) is equal to 0.3510 . The system $T$ will change from periodic motion to chaotic motion directly at $k_{1}=0.3510$ and then the phenomenon of intermittent chaos will appear.

Similar conclusion can be found in Fig.4(b). That is to say, when $k_{1}=0.3279$, the largest Lyapunov exponents corresponding to the system $T$ fluctuate around zero and the system $T$ is at quasi-periodic state. However, when the adjustment speed $k_{1}$ changes in the interval $(0.3356,0.3510)$, the corresponding largest Lyapunov exponents are less than zero, which means that the system $T$ is at periodic stable state. From Fig.4(b), a noticeable phenomenon is that there has been a dramatic change in the value of the largest Lyapunov exponent when $k_{1}=0.3510$, which may imply that the intermittent chaos will occur. Since this type of intermittency usually takes place along the boundary of periodic motion and chaotic motion, we can judge that PM-I type intermittent chaos appears when $k_{1}=0.3510$ according to the different conditions of PM-type intermittent chaos. This kind of intermittent chaos can lead to the transition from periodic motion to chaotic motion. The corresponding time series plot when $k_{1}=0.3510$ is shown in Fig.4(c). With the evolution of time, the system $T$ will transiently undergo the period-2 motion and then enter into chaotic state directly, when the PM-I type appears. It is found in the corresponding phase portrait that periodic-2 blue attractor and period-2 black chaotic attractor appear simultaneously, which is caused by intermittent chaos, as shown in Fig.4(d). 

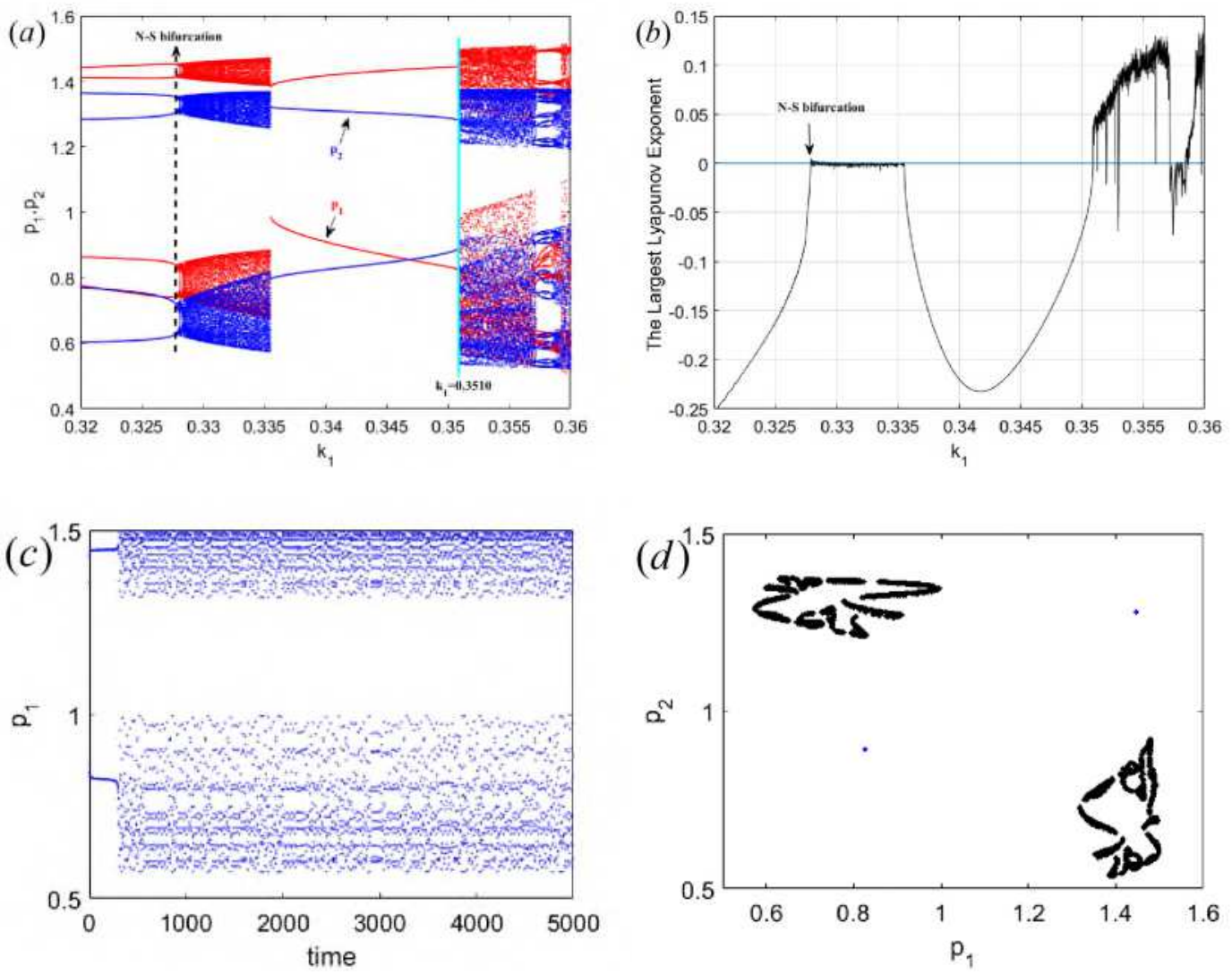

Fig.4. The parameters are fixed as $a=2.9147, b=2.8236, \quad \gamma=0.3552, \quad \theta=0.8802, \quad k_{2}=0.3744$, $s_{1}=0.8872, s_{2}=0.2240, c_{1}=0.8811$ and $c_{2}=0.7042$. (a) The bifurcation diagram in regard to decision parameter $k_{1}$, in which red represents the price $p_{1}$ of the manufacture $M_{1}$, blue represents the price $p_{2}$ of the manufacturer $M_{2}$, and the value of $k_{1}$ corresponding to the light blue line is 0.3510 . (b) The corresponding 1-D largest Lyapunov exponent diagram of bifurcation curves in Fig.4(a). (c) When $k_{1}=0.3510$, the corresponding time series plot of the system $T$. (d) When $k_{1}=0.3510$, the corresponding phase portrait of the system $T$, in which there is the phenomenon of coexistence between period-2 blue attractor and period-2 black chaotic attractor.

Keeping the other parameters unchanged, we further fix the value of $k_{1}$ as 0.3747 and choose the service level $s_{1}$ provided by the manufacturer $M_{1}$ as bifurcation parameter. The bifurcation diagram in regard to $s_{1}$ and its 1-D largest Lyapunov diagram are given in the first two figures of Fig.5, respectively. From Fig.5(a) and Fig.5(b), it can be clearly found that there are the phenomena of PM-III type intermittent chaos and crisis-induced intermittent chaos. In Fig.5(a), the bifurcation curves of $p_{1}$ and $p_{2}$ with respect to $s_{1}$ are respectively marked by red and blue. In addition, the values of $s_{1}$ at the three light blue lines are $s_{1}=0.7815, s_{1}=1.0050$ and $s_{1}=1.4010$. It is quite clear in Fig.5(b) that the system $T$ oscillates suddenly and sharply at $s_{1}=0.7815$. Furthermore, such special explosive mutations are also found at $s_{1}=1.0050$ and 
$s_{1}=1.4010$, which is related to the intermittent chaos occurring in the system $T$. The further discussions of PM-III intermittency and crisis-induced intermittency will be given by means of the time series plots and phase portraits, which are respectively exhibited in Fig.6 and Fig.7.
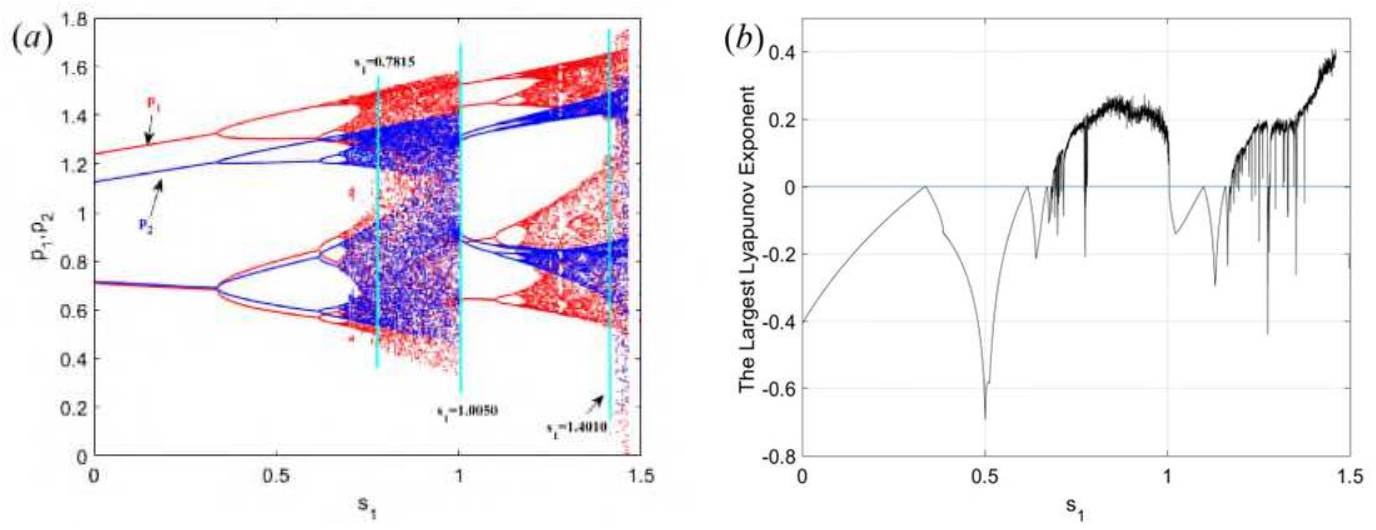

Fig.5. The parameters are chosen as $a=2.9147, b=2.8236, \gamma=0.3552, \quad \theta=0.8802, \quad k_{1}=0.3747$, $k_{2}=0.3744, s_{2}=0.2240, c_{1}=0.8811$ and $c_{2}=0.7042$. (a) The bifurcation diagram of $p_{1}$ and $p_{2}$ with respect to $s_{1}$, in which red denotes the price $p_{1}$ of the manufacturer $M_{1}$ and blue represents the price $p_{2}$ of the manufacturer $M_{2}$. In addition, the values of $s_{1}$ at three lines marked by light blue are $s_{1}=0.7815$, $s_{1}=1.0050$ and $s_{1}=1.4010$, respectively. (b) The corresponding 1-D largest Lyapunov exponent diagram of Fig.5(a).

It is similar to PM-I type intermittent chaos that PM-III type intermittency phenomenon also takes place along the boundary between periodic motion and chaotic motion, but a significant difference is that PM-III type intermittency phenomenon is generally due to subcritical flip bifurcation. When $s_{1}=0.7815$, the time series plot corresponding to the system $T$ and the phase portrait of two-piece of chaotic attractor are displayed in Fig.6(a) and Fig.6(b), respectively. In the time series plot, the time interval that the amplitude of state variable changes little is called laminar state, while the time interval in which the amplitude of state variable varies greatly is called burst state. On the whole, it can be distinctly found from Fig.6(a) that the laminar state and the burst state appear randomly and alternatively, and the burst state can break the laminar state at any time. Furthermore, the time of duration for the laminar state is stochastic, unpredictable and indefinite. The laminar state represents the periodic motion of the system $T$, while the burst state represents the chaotic motion. With gradual increase of the value of $s_{1}$, the PM-III type intermittent chaos will appear once again when $s_{1}=1.0050$. At this time, the corresponding time series plot and the phase portrait of one-piece of chaotic attractor are shown in Fig.6(c) and Fig.6(d), respectively. From this we can see that the alternative appearance of periodic motion state and chaotic motion state is a significant feature of PM-III intermittent chaos. 

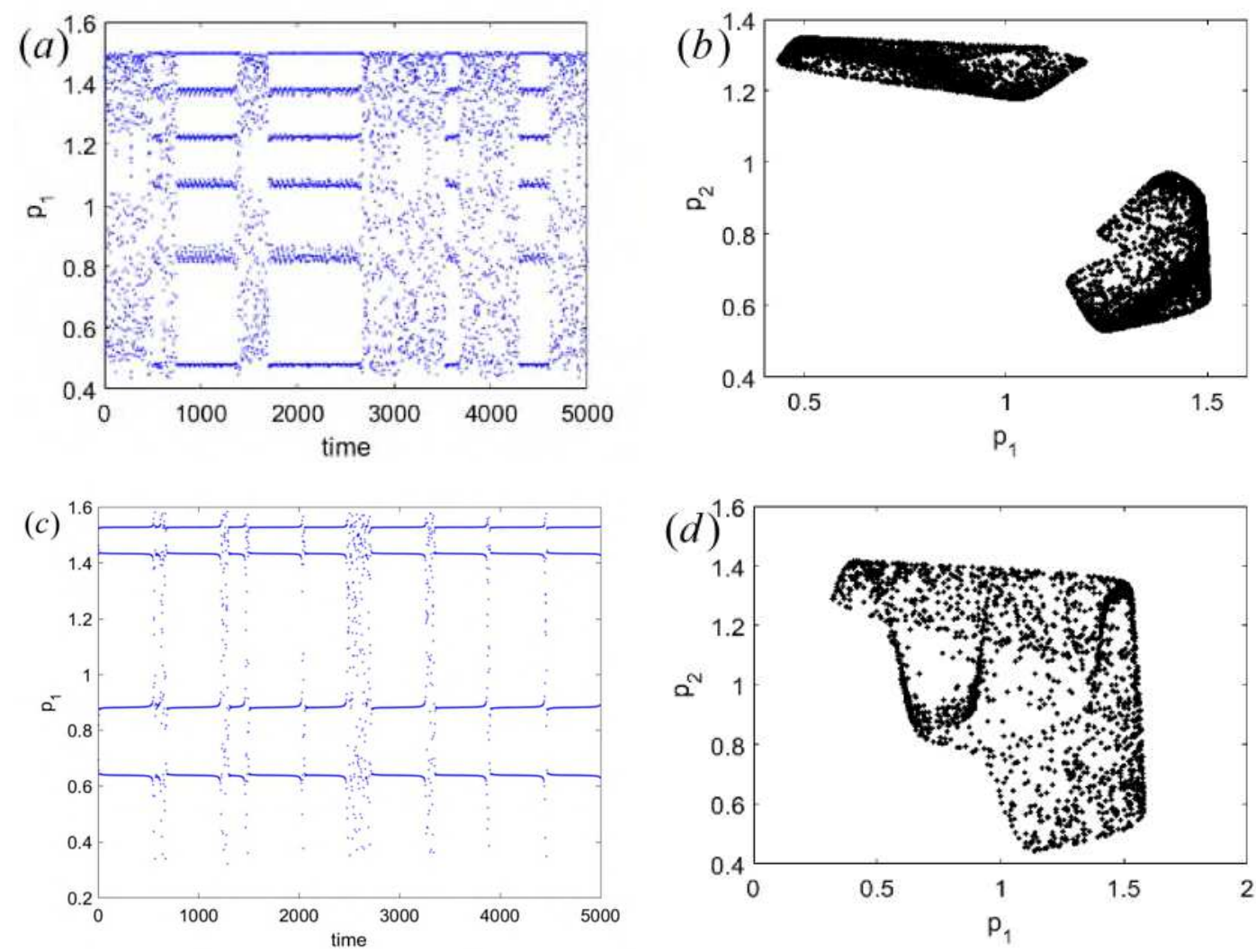

Fig.6. The parameter conditions are fixed as $a=2.9147, b=2.8236, \gamma=0.3552, \theta=0.8802, k_{1}=0.3747$, $k_{2}=0.3744, s_{2}=0.2240, c_{1}=0.8811$ and $c_{2}=0.7042$. (a) The time series plot, when $s_{1}=0.7815$. (b) When $s_{1}=0.7815$, the corresponding phase portrait of two-piece of chaotic attractor. (c) When $s_{1}=1.0050$, the time series plot. (d) When $s_{1}=1.0050$, the phase portrait of one-piece of chaotic attractor.

Another type of intermittent chaos existing in the system $T$ is crisis-induced intermittent chaos. The crisis is one kind of phenomenon that causing sudden change in the attractor, which is due to chaotic attractor collides with unstable or stable manifolds. It is subdivided into boundary crisis, inner crisis and attractor merging crisis. More in detail, the reason why inner crisis appears is that the attractor collides with the unstable periodic orbit in the attracting area of the attractor, which leads to sudden change in size of the attractor(a sudden expansion or reduction). However, attractor merging crisis is caused by that many attractors simultaneously collide with unstable periodic orbit in the attracting area of the attractor. As a result, the number of the attractor is reduced and multiple chaotic attractors will be merged into one-piece of chaotic attractor. In general speaking, the intermittent chaos caused by inner crisis and attractor merging crisis is called as crisis-induced intermittent chaos. In addition, we call a smaller chaotic attractor as a weak chaotic attractor.

Conversely, a slightly larger chaotic attractor is called a strong chaotic attractor. The phase portrait of two-piece of weak chaotic attractor of $T$ is shown in Fig.7(a), when $s_{1}=1.4010$. The corresponding time series plot is exhibited in Fig.7(b). With the evolution of time, two-piece of weak chaotic attractor will oscillate weakly in a limited range of the chaotic laminar states, but the motion of the system $T$ will not escape from the laminar states with limited range. However, with the increasing service level $s_{1}$ provided by $M_{1}$, there is the inner crisis occurs in the 
system $T$. As a result, the size of the attractor is further increased and then forming a strong chaotic attractor, as shown in Fig.7(c). Another notable phenomenon in Fig.7(d) is that strong chaotic laminar state could break weak chaotic laminar state ceaselessly and randomly, when the value of $s_{1}$ constantly increases to 1.4040. Subsequently, with further increase of the value of $s_{1}$ until $s_{1}=1.4440$, a strong chaotic attractor with large size and clear boundary is formed, as shown in Fig.7(e). Therefore, one remarkable characteristic of crisis-induced intermittent chaos is that in the corresponding time series plot, strong chaotic laminar state will smash the weak chaotic laminar state constantly and stochastically.
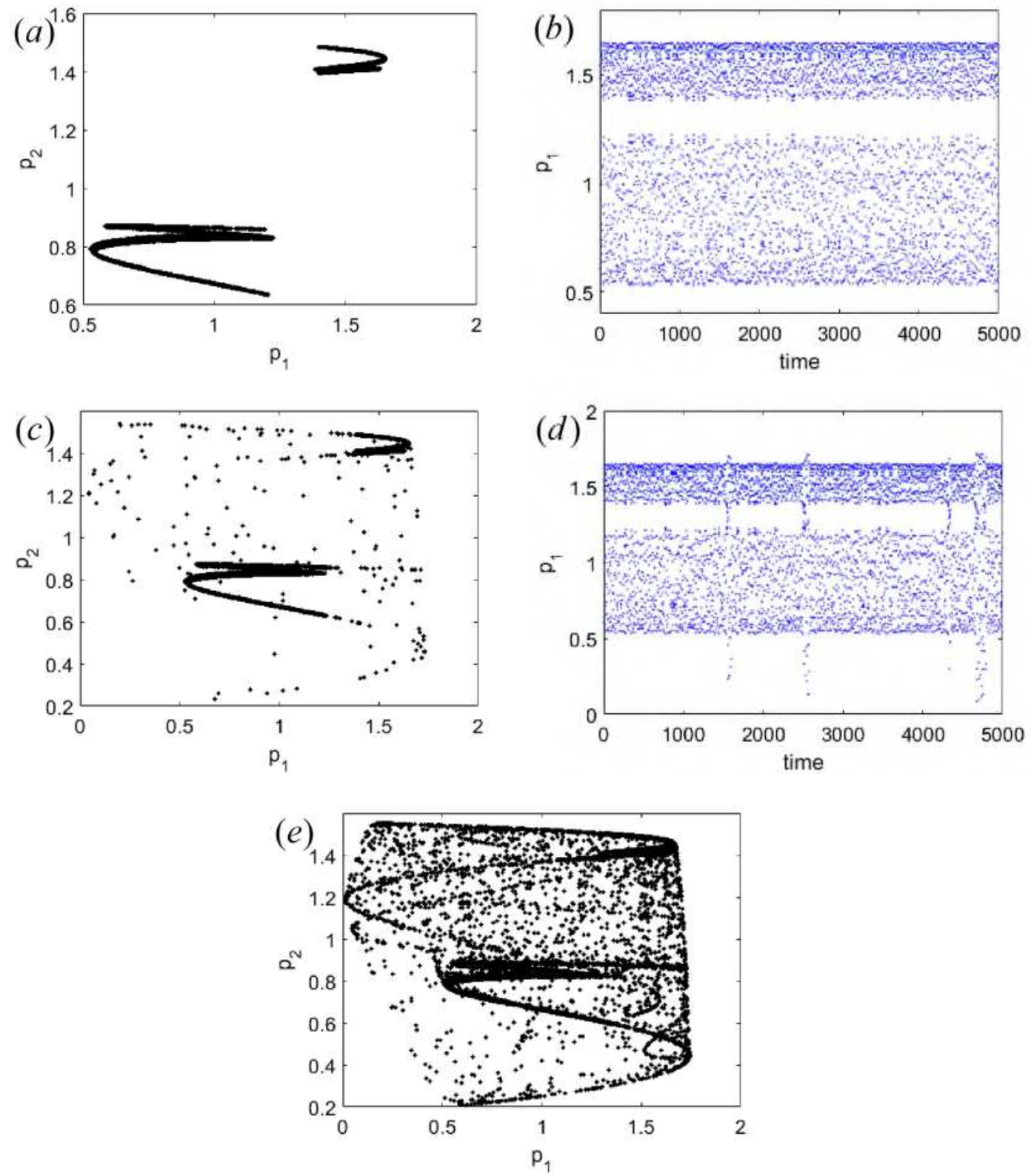

Fig.7. The parameter conditions are fixed as $a=2.9147, b=2.8236, \gamma=0.3552, \theta=0.8802, k_{1}=0.3747$, $k_{2}=0.3744, s_{2}=0.2240, c_{1}=0.8811$ and $c_{2}=0.7042$. (a) The phase portrait of two-piece of weak chaotic attractor, when $s_{1}=1.4010$. (b) The time series plot corresponding to the system $T$, when $s_{1}=1.4010$. (c) The phase portrait of the strong chaotic attractor, when $s_{1}=1.4040$. (d) The time series plot, when $s_{1}=1.4040$. (e) 
The strong chaotic attractor with clear boundary, when $s_{1}=1.4440$.

\section{Global dynamics}

The multi-stability of the system $T$ will be studied and analyzed in this section. Slight change of the value of parameter will not only change the number of solutions of the system $T$, but also cause the changes in the coexistence of attractors, the shape and structure of basin of attraction, and even a gap will appear in boundary of basin of attraction. Next, we will discuss the multi-stability by means of attracting basins and critical curves. First of all, the definitions of an invariant set and an attractor are given as follows,

Definition 1. A set $\mathrm{A}$ belonged to $R^{n}$ is an invariant set of a mapping $\mathrm{T}$, if the set $\mathrm{A}$ can be mapped to itself. That is to say, $\mathrm{T}(\mathrm{A})=\mathrm{A}$.

According to the definition of the invariant set, we can know that if there is a point satisfy $p \in \mathrm{A}$, then we have $\mathrm{T}(p) \in \mathrm{A}$. And the invariant set $\mathrm{A}$ is also an attractor of the mapping $\mathrm{T}$, when the conditions in Definition 2 below hold.

Definition 2. A closed invariant set $A$ is an attractor of a mapping $T$, if one of the following two conditions holds,

(i) The closed invariant set $\mathrm{A}$ is stable in the sense of Lyapunov;

(ii) There is a neighborhood $\Psi$ of the closed invariant set $\mathrm{A}$ exists, for $\forall\left(p_{1}, p_{2}\right) \in \Psi$, when

$$
t \rightarrow \infty \text {, then } \mathrm{T}^{t}\left(p_{1}, p_{2}\right) \rightarrow \mathrm{A}
$$

Furthermore, the basin of attraction $\mathrm{B}(\mathrm{A})$ corresponding to the attractor $\mathrm{A}$ can be given as

$$
\mathrm{B}(\mathrm{A})=\left\{\left(p_{1}, p_{2}\right) \mid \mathrm{T}^{t}\left(p_{1}, p_{2}\right) \rightarrow \mathrm{A}, t \rightarrow \infty\right\} .
$$

In other words, the basin of attraction $\mathrm{B}(\mathrm{A})$ of the attractor $\mathrm{A}$ includes all of trajectories that converge to $\mathrm{A}$. A more general form of $\mathrm{B}(\mathrm{A})$ is given below,

$$
\mathrm{B}(\mathrm{A})=\bigcup_{t=0}^{\infty} \mathrm{T}^{-t}(\Psi(\mathrm{A})) .
$$
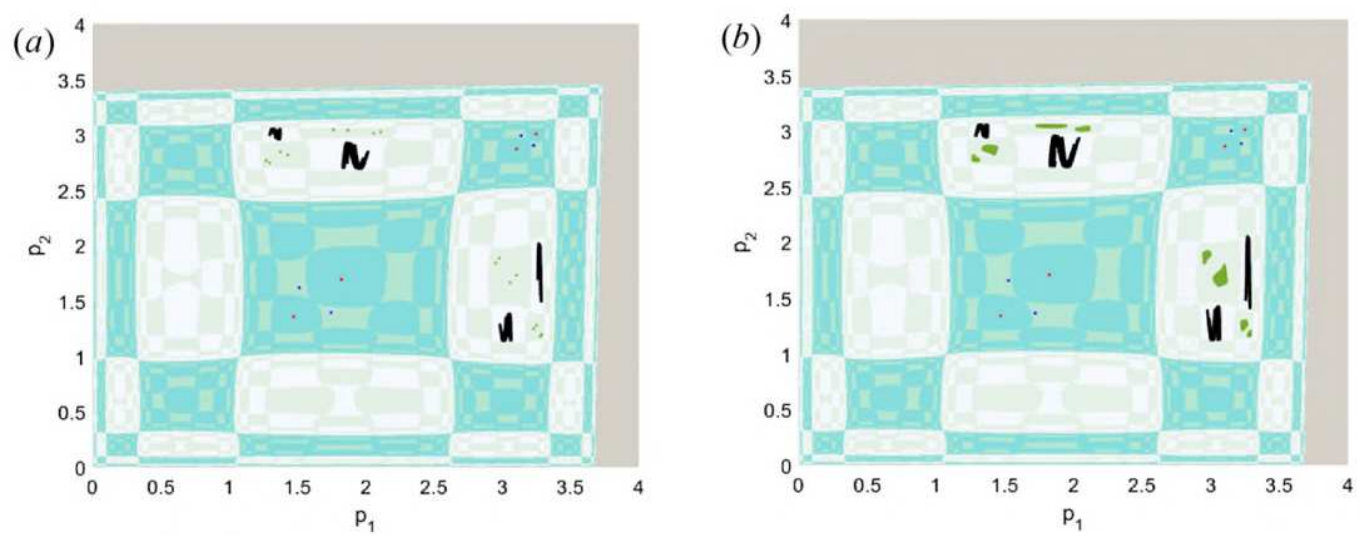

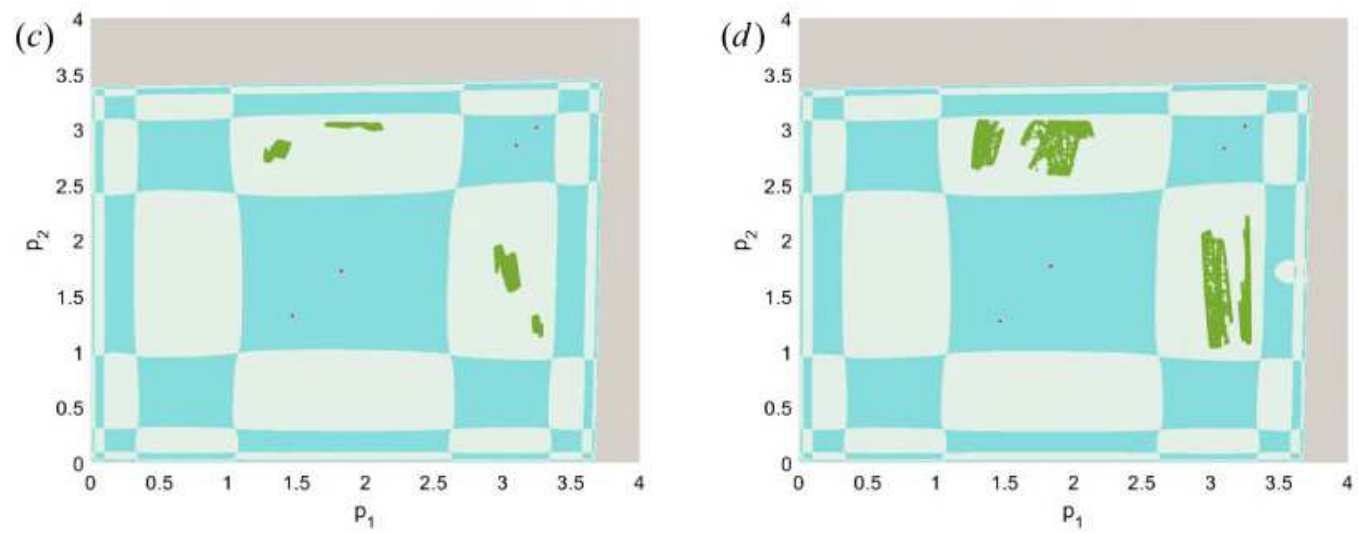

Fig.8. A group of basins of attraction, in which the parameter conditions are $a=3.2636, b=1.7114$, $\gamma=0.0566, \theta=0.4204, k_{1}=0.2765, s_{1}=2.4853, s_{2}=2.9951, c_{1}=1.1472$ and $c_{2}=0.5702$. Different attractors are marked by different colors and have their own attracting areas. In addition, the infeasible region of the system $T$ is marked by gray. (a) $k_{2}=0.3000$. (b) $k_{2}=0.3010$. (c) $k_{2}=0.3020$. (d) $k_{2}=0.3055$.

\subsection{Coexistence of multiple attractors}

A set of parameters is fixed as $a=3.2636, b=1.7114, \gamma=0.0566, \theta=0.4204, k_{1}=0.2765$, $k_{2}=0.3000, s_{1}=2.4853, s_{2}=2.9951, \quad c_{1}=1.1472$ and $c_{2}=0.5702$. Under this set of parameter condition, the corresponding basin of attraction of the system $T$ is shown in Fig.8(a). It is obvious from Fig.8(a) that there are three groups of periodic attractors and one group of chaotic attractors coexisting in basin of attraction, and each group of attractors has its corresponding attracting area. In addition, the infeasible region of system $T$ is marked by gray. Keeping the other parameters unchanged and increasing the value of $k_{2}$ slightly to 0.3010 , the corresponding basin of attraction is given in Fig.8(b). No significant changes in the shape and structure of the basin of attraction, but the green periodic attractors become chaotic attractors. In addition, the type of coexistence of attractors in basin of attraction also changes, that is, two groups of chaotic attractors coexist with two groups of periodic attractors. It is also obvious in Fig.8(b) that with constant increase of $k_{2}$, the size of black chaotic attractors grows up obviously and the blue periodic attractors and black chaotic attractors will contact with their border of attracting region soon, and then the "boundary crisis" [41] will be induced.

The blue periodic attractors and black chaotic attractors will disappear completely after contacting the boundary of their attracting area, when $k_{2}=0.3020$. That is, a contact bifurcation [25-26] occurs in the system $T$. There are only two groups of attractors coexisting in the basin of attraction at this time, that is, red periodic attractors and green chaotic attractors, as shown in Fig.8(c). Furthermore, when the value of $k_{2}$ increases to 0.3055 , the corresponding basin of attraction of the system $T$ can be seen in Fig.8(d), in which there is no changes in the type of coexistence of attractors, the shape and structure of basin of attraction. However, the size of green 
chaotic attractors significantly becomes bigger. As a result, if $M_{2}$ changes its product pricing decisions blindly and frequently, it will not only disturb the market competition order, but also increase the unpredictability of market competition, and even lead to the market competition into an irreversible chaotic state.


Fig.9. The parameter conditions are fixed as $a=2.8062, b=2.0579, \gamma=0.0604, \theta=0.5004, s_{1}=1.5527$, $s_{2}=1.5527, c_{1}=1.5189$ and $c_{2}=1.5189$. (a) $k_{1}=0.2994, k_{2}=0.2994$. (b) $k_{1}=0.3094, k_{2}=0.3094$.

There are four groups of attractors coexisting in the basin of attraction when the parameter conditions are fixed as $a=2.8062, b=2.0579, \gamma=0.0604, \theta=0.5004, s_{1}=1.5527$, $s_{2}=1.5527, c_{1}=1.5189$ and $c_{2}=1.5189$. Different attractors have their own attracting areas and the infeasible region of system $T$ is marked by gray. As shown in Fig.9(a), there is one group of period-4 red attractor located on the diagonal and another three groups of periodic attractors arranged symmetrically on both sides of the diagonal, in which the adjustment speeds of $M_{1}$ and $M_{2}$ are given as $k_{1}=0.2994$ and $k_{2}=0.2994$. With the value of $k_{1}$ and $k_{2}$ slightly increase to $k_{1}=0.3094$ and $k_{2}=0.3094$, two groups of period-8 attractor symmetrically distributed on both sides of the diagonal will first evolve into period-4 chaotic attractors, and finally the period-4 chaotic attractors will become bigger and then merge into two pieces of chaotic attractors, as shown in Fig.9(b). From Fig.9(b), it also can be clearly seen that the period-4 black attractor has been evolved into period-8 black chaotic attractor. With further increase of $k_{1}$

and $k_{2}$, there may have three groups of chaotic attractors coexisting in the basin of attraction.

\subsection{Critical curves}

In the next evolution process of two groups of basin of attraction, by comparing with the previous basins of attraction, it can be clearly find that there are significant changes in the boundary and interior of the basin of attraction. That is, there are "gaps" or "holes" appear on the boundary and interior of basin of attraction. A reasonable conjecture will be proposed that these changes in the boundary and interior of the basin of attraction may be related to the global bifurcation with 
gradual changes in parameters. In addition, with the help of the critical curves, the sudden changes in qualitative properties, like the shape and structure of these basins of attraction, can be further studied. The mapping $T$ is an invertible mapping, since taking any point $\left(p_{1}, p_{2}\right)$ from $R^{2}$ plane arbitrarily, we can get a unique point $\left(p_{1}^{\prime}, p_{2}{ }^{\prime}\right)$ after mapping $T$, while from the inverse mapping of the mapping $T$ cannot uniquely determine a preimage point. Furthermore, from calculating a fourth degree algebraic system (7) with respect to $p_{1}$ and $p_{2}$, we can get four preimage points of the origin point $O=(0,0)$. It is obvious that the origin $O=(0,0)$ itself is a preimage point. In addition, there are two other preimage points located on two positive coordinates, that is, $O_{-1}^{(1)}=\left(0, \frac{B}{2 b}\right)$ and $O_{-1}^{(2)}=\left(\frac{A}{2 b}, 0\right)$. By combining the following two equations

$$
\left\{\begin{array}{l}
1+k_{1}\left(a+s_{1}+\theta s_{2}+b c_{1}\right)-2 b k_{1} p_{1}+k_{1} \gamma p_{2}=0 \\
1+k_{2}\left(a+s_{2}+\theta s_{1}+b c_{2}\right)-2 b k_{2} p_{2}+k_{2} \gamma p_{1}=0
\end{array},\right.
$$

the last preimage point is $O_{-1}^{(3)}=\left(\frac{2 b k_{2}+2 b k_{1} k_{2} A+k_{1} \gamma+k_{1} k_{2} \gamma B}{k_{1} k_{2}\left(4 b^{2}-\gamma^{2}\right)}, \frac{2 b k_{1}+2 b k_{1} k_{2} B+k_{2} \gamma+k_{1} k_{2} \gamma A}{k_{1} k_{2}\left(4 b^{2}-\gamma^{2}\right)}\right)$.

The preimage $L C_{-1}$ of the critical curves $L C$ is a locus formed by the points whose determinant of Jacobian matrix (9) corresponding to the system $T$ is zero. After some simple calculations, two preimages $L C_{-1}^{(a)}$ and $L C_{-1}^{(b)}$ of the critical curves of the system $T$ can be respectively given as following,

$$
L C_{-1}^{(a)}=\frac{-n-\sqrt{n^{2}-64 k_{1} k_{2} b \gamma\left(1+k_{2} B+k_{1} A+k_{1} k_{2} A B+\left(m-4 k_{1} k_{2} \gamma b p_{2}\right) p_{2}\right.}}{32 k_{1} k_{2} b \gamma}
$$

and

$$
L C_{-1}^{(b)}=\frac{-n+\sqrt{n^{2}-64 k_{1} k_{2} b \gamma\left(1+k_{2} B+k_{1} A+k_{1} k_{2} A B+\left(m-4 k_{1} k_{2} \gamma b p_{2}\right) p_{2}\right.}}{32 k_{1} k_{2} b \gamma},
$$

in which $m$ and $n$ are auxiliary variables introduced for simple calculation, $m=k_{1} \gamma+k_{1} k_{2} \gamma B-4 k_{2} b-4 k_{1} k_{2} A b$ and $n=k_{2} \gamma+k_{1} k_{2} \gamma A-4 k_{1} b-4 k_{1} k_{2} B b$. The corresponding two critical curves can be obtained after the mapping $T$. That is, $T\left(L C_{-1}^{(a)}\right)=L C^{(a)}$ and $T\left(L C_{-1}^{(b)}\right)=L C^{(b)}$. Moreover, two critical curves $L C^{(a)}$ and $L C^{(b)}$ of the system $T$ divide the two-dimensional phase plane into the three areas of $Z_{0}-Z_{2}-Z_{4}$ type, in which the $Z_{0}$ area represents that the points in this area have no corresponding preimage points, the $Z_{2}$ area represents that the points in this area have two different preimages. Analogically, the points in $Z_{4}$ 
area have four different preimages. It should be noted that the origin point $O=(0,0)$ must be in $Z_{4}$ area.

The case of three groups of attractors coexisting in the basin of attraction is displayed in Fig.10. The parameter conditions are $a=1.1673, b=0.7101, \gamma=0.5302, \theta=0.9303$, $k_{2}=0.4139, s_{1}=0.7033, s_{2}=0.6205, c_{1}=0.1121$ and $c_{2}=1.9901$. As shown in Fig.10(a), when $k_{1}=0.2900$, there are the phenomenon of coexistence between three groups of attractors. The attracting areas of these three groups of attractors are denoted as brown, yellow and green, respectively. The dark blue region is the infeasible region. In addition, an obvious gap appears on right boundary of attracting basin. The corresponding basin of attraction is shown in Fig.10(b), when the value of $k_{1}$ increases to 0.2912 slightly. No changes in the type of coexistence of attractors, and there are still three groups of attractors coexisting in the basin of attraction. However, period- 8 red attractor becomes period- 8 chaotic attractor, and period-36 black attractor becomes period-18 chaotic attractor. From Fig.10(b), we can also see that the border of black attractors will almost get in touch with their boundaries of green attracting area, and the size of brown attracting area corresponding to red attractors grows significantly.

In Fig.10(c)-Fig.10(f), the boundary of basin of attraction are marked by white lines on two-dimensional phase plane, the preimages $L C_{-1}^{(a)}$ and $L C_{-1}^{(b)}$ of critical curves of the system $T$ are denoted by bright blue, and the critical curves $L C^{(a)}$ and $L C^{(b)}$ are marked by pink curves. With $k_{1}$ increases to 0.2916 little by little, we can find that the boundary of black chaotic attractors will get in touch with borders of their attracting areas. Then the system $T$ may have a contact bifurcation. Then the black chaotic attractors and their green attracting area disappear completely, as shown in Fig.10(c). At this time, only red chaotic attractors coexist with blue periodic attractors in basin of attraction. It can be seen in Fig.10(c) that the upper end of the critical curve $L C^{(b)}$ crosses the upper boundary $O_{-1}^{(1)} O_{-1}^{(3)}$ of the basin of attraction, while the lower end of $L C^{(b)}$ is still included inside the basin of attraction. When further increasing the value of $k_{1}$ to $k_{1}=0.3066$, the basin of attraction of the system $T$ is shown in Fig.10(d). Compared with Fig.10(c), period-8 red chaotic attractor becomes period-18 red chaotic attractor, and its brown attracting area becomes smaller. The boundary of red chaotic attractors almost contacts with the boundary of brown attracting area. From Fig.10(d), another notable change is that the lower end of critical curve $L C^{(b)}$ slightly crosses the right border $O_{-1}^{(2)} O_{-1}^{(3)}$ of basin of attraction. There is a small gap appears in the upper boundary of basin of attraction.

When $k_{1}=0.3594$, there is only one homoclinic cycle existing in the basin of attraction, as shown in Fig.10(e). Furthermore, the lower end of critical curve $L C^{(b)}$ moves to the right greatly, and the gap on the upper boundary of the basin becomes larger. With constant increase of the 
value of $k_{1}$, the size of blue homoclinic cycle gets bigger gradually. Until $k_{1}=0.3874$, the lower end of $L C^{(b)}$ further moves to the right and the size of blue homoclinic cycle significantly increases. The boundary of homoclinic cycle almost contacts with the border of attracting basin and critical curve $L C^{(b)}$. With further increasing of $k_{1}$, the final bifurcation [25-26] will appear in the system $T$, and then the homoclinic cycle will disappear after rupture. However, the skeleton of its yellow attracting area will still exist in the inner of basin of attraction, which is consisted by dense scattered points. This is the so-called ghost phenomenon.
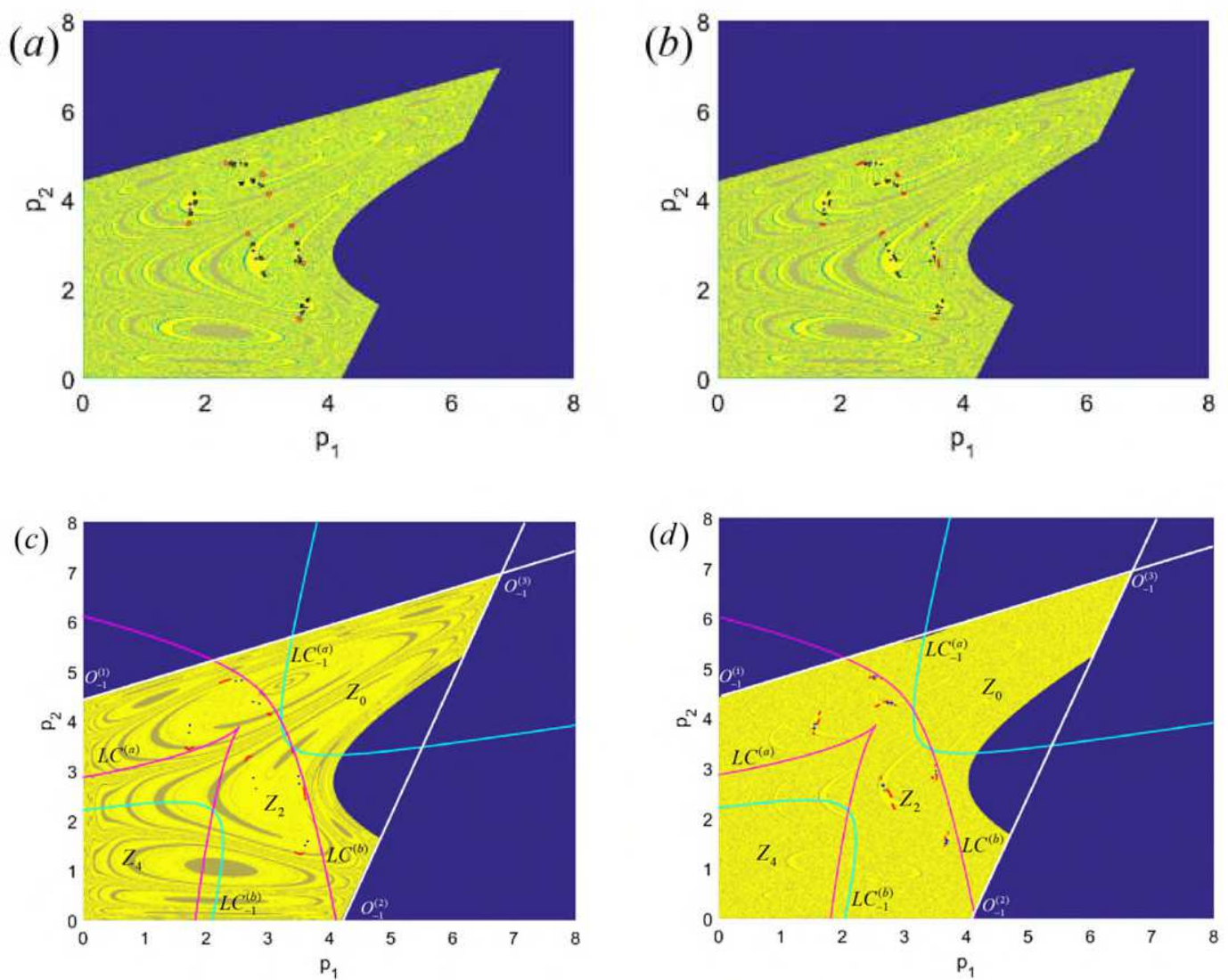

(e)
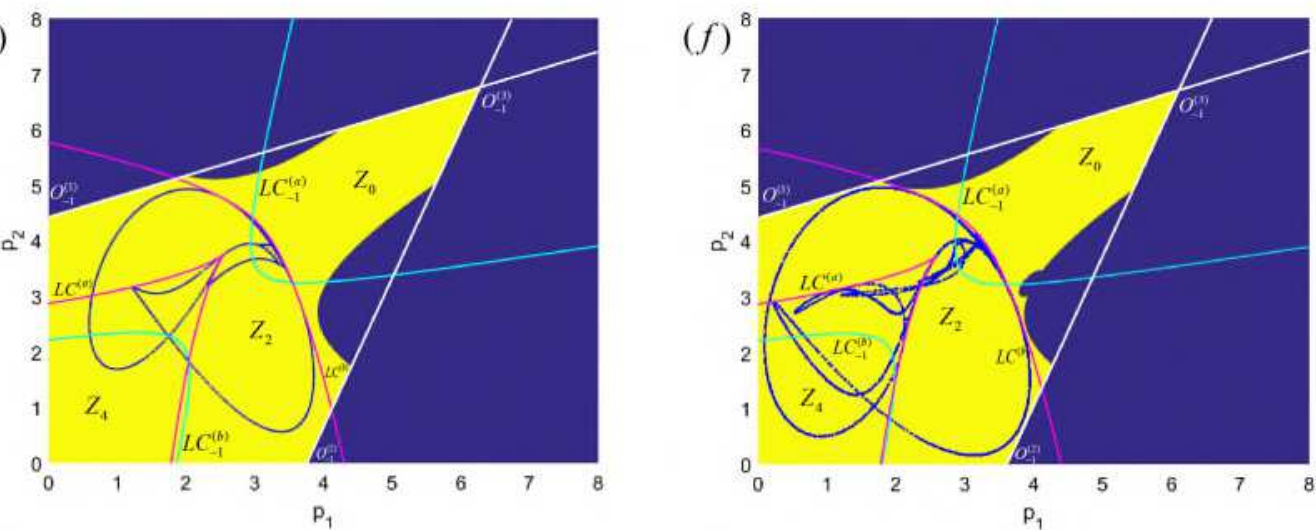

Fig.10. The parameters are fixed as $a=1.1673, b=0.7101, \quad \gamma=0.5302, \quad \theta=0.9303, \quad k_{2}=0.4139$, $s_{1}=0.7033, s_{2}=0.6205, c_{1}=0.1121$ and $c_{2}=1.9901$. The corresponding attracting areas of red, blue and black attractors are respectively marked by three different colors, that is, brown, yellow and green. In addition, the 
infeasible region is marked by dark blue. The borders of basin of attraction are denoted by white lines, the preimages $L C_{-1}^{(a)}$ and $L C_{-1}^{(a)}$ of critical curves are marked by bright blue and critical curves $L C^{(a)}$ and $L C^{(b)}$ of system $T$ are marked by pink. (a) $k_{1}=0.2900$. (b) $k_{1}=0.2912$. (c) $k_{1}=0.2916$. (d) $k_{1}=0.3066$. (e) $k_{1}=0.3594$. (f) $k_{1}=0.3874$.

The evolution processes of a group of basin of attraction with respect to the decision adjustment speeds $k_{1}$ and $k_{2}$ are given in Fig.11(considering the situation that $k_{2}=k_{1}$ ), the corresponding parameter conditions are chosen as $a=3.5663, b=3.4499, \quad \gamma=0.9199$, $\theta=0.9695, s_{1}=0.2844, s_{2}=0.2844, c_{1}=2.4196$ and $c_{2}=2.4196$. As shown in Fig.11(a) that there are two groups of attractors coexisting in the basin of attraction when $k_{1}=0.1600$. That is to say, red periodic attractors(whose attracting region is marked by blue) coexist with blue periodic attractors(whose corresponding attracting area is yellow area). However, when $k_{1}=0.1607$, the number of coexisting attractors in the basin of attraction increases from 2 to 4 , as shown in Fig.11(b). The period-6 red attractor and period-6 blue attractor are both changed into periodic-6 red invariant cycle and periodic-6 blue invariant cycle. These two different colors invariant cycles are surrounded by green periodic attractors and pink periodic attractors, respectively. These four groups of different attractors have their respective attracting areas, which are marked by different colors in Fig.11(b). Increasing the value of $k_{1}$ slightly to 0.1609, the type of coexisting attractors in the basin of attraction has changed significantly again. From Fig.11(c), it can be clearly seen that only red chaotic attractors coexist with blue chaotic attractors in basin of attraction, whose corresponding attracting areas are marked by blue and yellow respectively. Furthermore, it is quite obvious that the border of red chaotic attractors will almost get in touch with the boundary of their blue attracting area.

In Fig.11(d)-Fig.11(f), the borders of attracting basin are marked by white lines on two-dimensional phase plane, the preimages $L C_{-1}^{(a)}$ and $L C_{-1}^{(b)}$ of critical curves of the system $T$ are denoted by pink, and the critical curves $L C^{(a)}$ and $L C^{(b)}$ are marked by green. As the value of $k_{1}$ increases gradually, red chaotic attractors and their corresponding blue attracting area will disappear together. In Fig.11(d), when $k_{1}=0.1650$, there is two-piece of blue chaotic attractors existing in attracting basin. Both two ends of the critical curve $L C^{(b)}$ are included in the inner of the basin of attraction and no gap appears in the boundary of basin. However, when the value of $k_{1}$ increases to 0.1770 , the size of two-piece of chaotic attractors gradually grows up until one-piece of blue chaotic attractor is formed. It can be clearly seen from Fig.11(e) that the two ends of critical curve $L C^{(b)}$ cross the upper boundary $O_{-1}^{(1)} O_{-1}^{(3)}$ and right border $O_{-1}^{(2)} O_{-1}^{(3)}$ of the basin of attraction, respectively. There are obvious gaps appear in both the upper boundary and right border of attracting basin. In addition, the boundary of blue chaotic attractors not only will 
get in touch with a branch $L C^{(b)}$ of critical curves, but also will get in touch with the border of attracting basin soon. The size of blue chaotic attractor grows up as further increase of the parameter $k_{1}$. Until its boundary contacts with the boundary of basin of attraction, the blue chaotic attractor will disappear after rupture. Then there are holes appear inside the basin of attractor, and the new blue periodic attractor will appear and occupy the yellow attracting area corresponding to the original chaotic attractor. As shown in Fig.11(f), when $k_{1}=0.1974$, the situation that two groups of attractors coexist in basin of attraction appears again. At this time, blue periodic attractors coexist with red periodic attractors and the corresponding attracting areas are marked by yellow and blue. Besides, there are not only many gaps appear on the borders of attracting basin, but also many holes of different sizes in the interior of the basin of attraction.
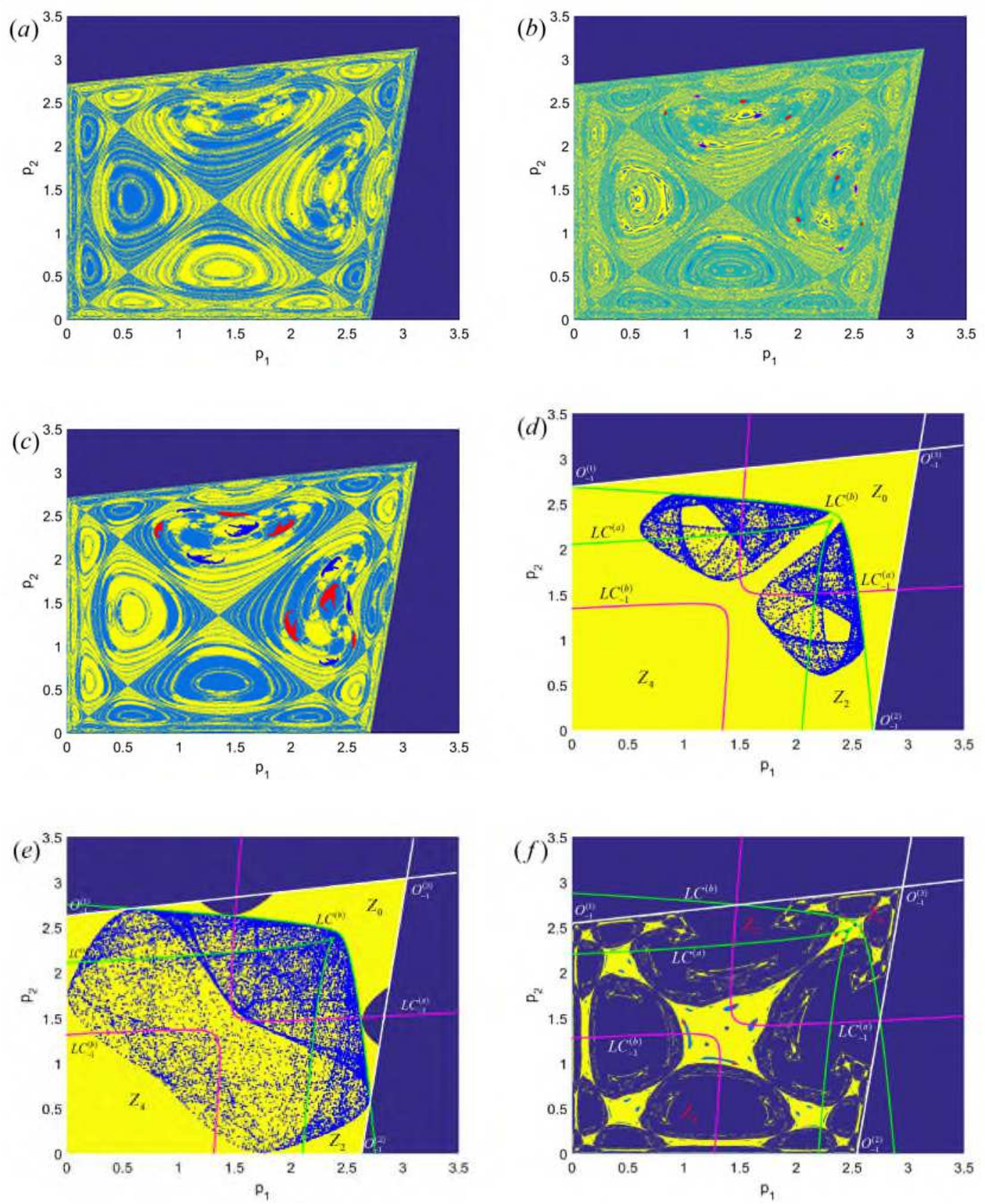
Fig.11. The evolution processes of basin of attraction for the system $T$ with respect to the decision adjustment speeds $k_{1}$ and $k_{2}$ (considering the situation that $k_{2}=k_{1}$ ). The parameters are fixed as $a=3.5663, b=3.4499$, $\gamma=0.9199, \theta=0.9695, s_{1}=0.2844, s_{2}=0.2844, c_{1}=2.4196$ and $c_{2}=2.4196$. The borders of basin of attraction are marked by white line, the two preimages $L C_{-1}^{(a)}$ and $L C_{-1}^{(b)}$ of the critical curves are marked by pink and two critical curves $L C^{(a)}$ and $L C^{(b)}$ are denoted by green. (a) $k_{1}=0.1600$. (b) $k_{1}=0.1607$. (c) $k_{1}=0.1609$. (d) $k_{1}=0.1650$. (e) $k_{1}=0.1770$. (f) $k_{1}=0.1974$.

\section{Profit analyses with different market environments}

The parameter conditions are chosen as $a=4.9196, b=1.2998, k_{1}=0.1676, k_{2}=0.1675$, $s_{1}=2.0513, s_{2}=2.4338, c_{1}=0.2287, c_{2}=1.1081$ and $h=0.1$. The price bifurcation diagram in three-dimensional parameter $\left(\theta, \gamma, p_{1}\right)$-space is shown in Fig.12(a). From Fig.12(a), we can know that with increase of $\theta$ and $\gamma$, the price of the products produced by $M_{1}$ becomes higher and higher. Fig.12(b) is the profit bifurcation diagram of $M_{1}$ in $\left(\theta, \gamma, \pi_{1}\right)$-space. The larger the values of $\theta$ and $\gamma$ are, the more unpredictable the manufacturer's profit is. The contour map in the bottom of Fig.12(b) is displayed separately in Fig.12(c), which implies that the profits $\pi_{1}$ of $M_{1}$ on the same isoline are equal. Moreover, the isolines of different colors correspond to different values of $\pi_{1}$. The heat map related to the revenue of $M_{1}$ is shown in Fig.12(d). From Fig.12(d), it can be known that when the parameters $\theta$ and $\gamma$ remain in a relatively small range and then gradually increasing their values, the profit of $M_{1}$ will increase little by little. As shown in Fig.12(d), when the parameters $\theta$ and $\gamma$ remain in a relatively small range, the colors corresponding to the profit of $M_{1}$ are smooth, regular and orderly. With the values of $\theta$ and $\gamma$ increase, the colors corresponding to $\pi_{1}$ gradually change from green to blue. However, with constant increase of the parameters $\theta$ and $\gamma$, the system will become unpredictable and confused.

The last two figures in Fig.12 are two profit bifurcation diagrams with respect to the service spillover effect $\theta$ and the product differentiation $\gamma$, which are shown in Fig.12(e) and Fig.12(f), respectively. In addition, the color red represents $M_{1}$ and blue represents $M_{2}$. From Fig.12(e), when $\gamma=0.6907$, with the value of $\theta$ changes, it is clear that both equilibrium profit and average profit of $M_{1}$ are always higher than those of $M_{2}$. Moreover, the equilibrium profits of the two manufacturers are greater than their average profits. However, as the value of $\theta$ increases gradually, the system $T$ will enter into chaos irreversibly. Then the situation that the average profit of $M_{1}$ is less than the equilibrium profit of $M_{2}$ will occur. 
The profit bifurcations of two manufacturers with respect to $\gamma$ can be seen in Fig.12(f), where $\theta=0.5967$. We can know form Fig.12(f) that the equilibrium profit and average profit of the two manufacturers are equal when $\gamma<0.3$. However, with further increase of $\gamma$, the average profits of two manufacturers will be lower than their respective equilibrium profits. On the whole, both the equilibrium profit and average profit of $M_{1}$ are slightly higher than those of $M_{2}$, while the situation may appear when the system $T$ enters into chaos as the value of $\gamma$ gradually increases. That is, the average profit of $M_{1}$ will be lower than the equilibrium profit of $M_{2}$. Therefore, we can conclude that the values of product differentiation degree and service spillover effect are not the bigger the better.

(a)

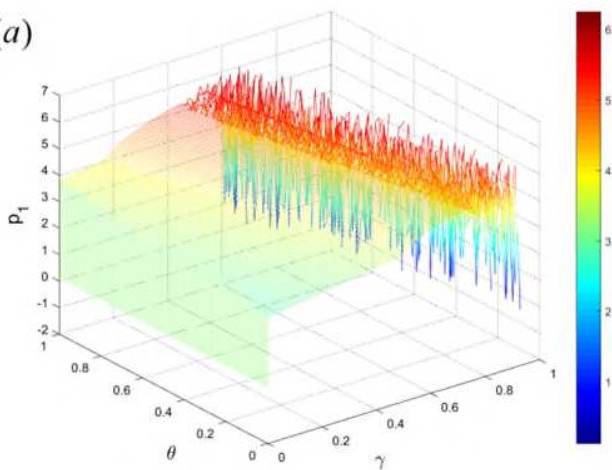

(c)

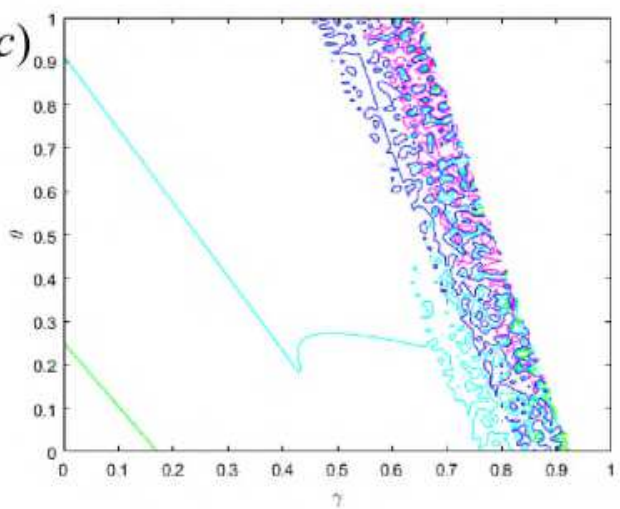

(e)

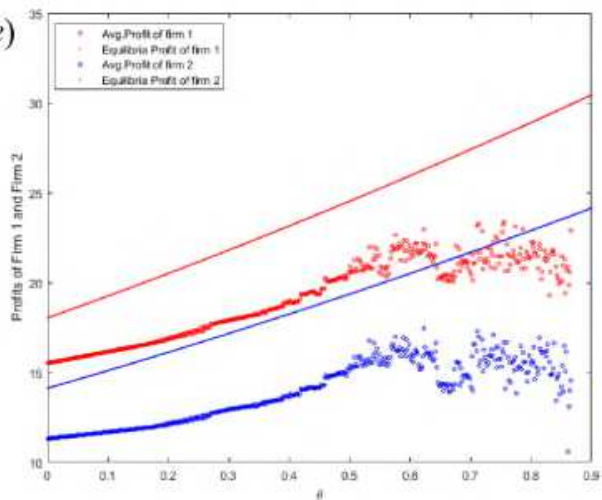

(b)

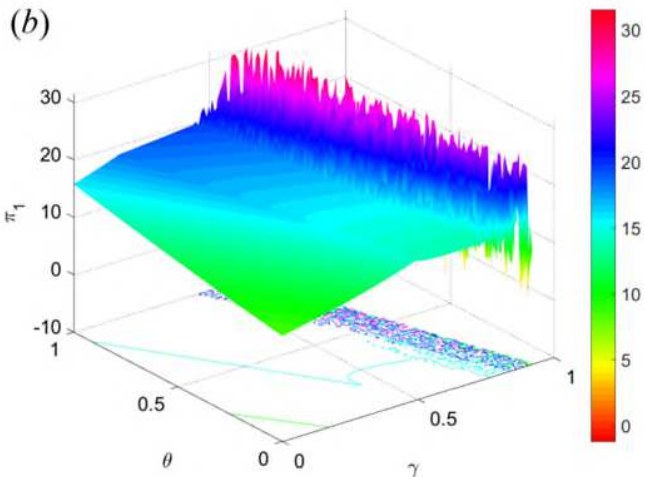

(d)

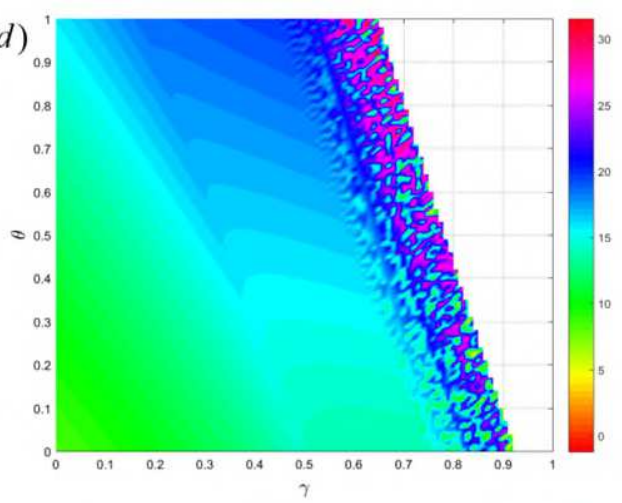

(f

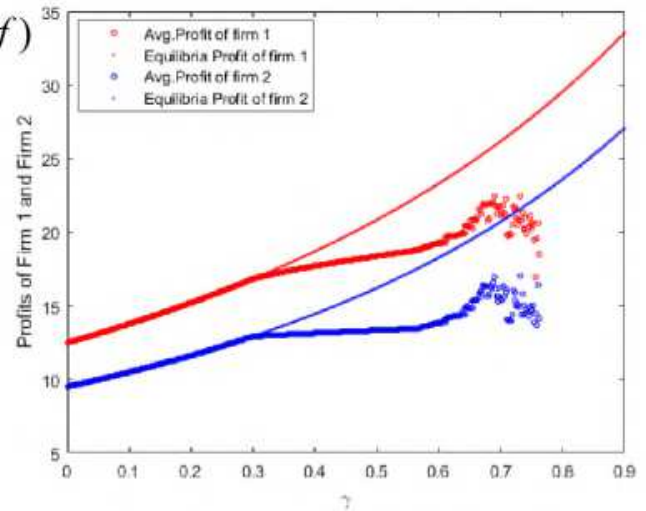

Fig.12. The parameter conditions are fixed as $a=4.9196, b=1.2998, k_{1}=0.1676, k_{2}=0.1675, s_{1}=2.0513$, $s_{2}=2.4338, c_{1}=0.2287, c_{2}=1.1081$ and $h=0.1$. (a) The price bifurcation diagram of $M_{1}$ in $\left(\theta, \gamma, p_{1}\right)$ 
-space. (b) The profit bifurcation diagram of $M_{1}$ in $\left(\theta, \gamma, p_{1}\right)$-space. (c) The contour map in $(\gamma, \theta)$-plane. (d)

The heat map $(\gamma, \theta)$-plane. (e) $\gamma=0.6907$. (f) $\theta=0.5967$.

\section{Conclusions}

In this research, we first analyze a static duopolistic Bertrand game with taking differentiated products and service spillover effect into consideration. It is concluded that there is a unique Nash equilibrium exists. By using the gradient mechanism, a dynamic game model of duopoly Bertrand is established. The local stability analyses on the four equilibrium solutions can be further obtained with the help of the knowledge of nonlinear dynamics and the Jury criterion. It can be known that both three boundary equilibrium points are unstable. At Nash equilibrium point, the system only can lose its stability through flip bifurcation.

In the part of numerical simulation, we can see from the 1-D bifurcation diagrams that there are two paths to chaos, that is, flip bifurcation and Neimark-Sacker bifurcation. Both the parameters that decision adjustment speeds, service levels, service spillover effect and price sensitivity coefficient do have significant impacts on steady properties of the system. In addition, we have further studied and analyzed a special case, and then we have found that there are many symmetric structures in $\left(b_{1}, b_{2}\right)$-plane. Another type of bifurcation, saddle-homoclinic bifurcation, will be observed during the evolution process of phase portraits of the attractors. With the change of the parameter, two foci with rough edge will finally evolve to a homoclinic cycle via the saddle-homoclinic bifurcation.

In addition to the above three bifurcation types, the phenomenon of intermittent chaos in the system is also a special dynamic behavior worthy of study and analysis. The emergence of intermittent chaos implies that this established economy system has good capability of self-regulating. In 1-D bifurcation diagram and the 1-D largest Lyapunov exponent diagram, it can be found that the amplitude of state variable will change abruptly at a certain time. This explosive change is related to the intermittent chaos of the system. Three different kinds of intermittency phenomenon have been discussed in details, namely, PM-I intermittency, PM-III intermittency and crisis-induced intermittency. Through the analysis, we can know that PM-I intermittency and PM-III intermittency occur along the border of periodic motion and chaotic motion transition. Moreover, PM-I intermittent chaos can lead to the system from periodic motion to chaotic motion. However, PM-III intermittent chaos is generally related to subcritical flip bifurcation. In time series plot, one of the main characteristics of crisis-induced intermittent chaos is that strong chaotic laminar state can destroy and break weak chaotic laminar state constantly and stochastically.

Moreover, it is found that the change of the parameters will not only change the shape, size and number of coexisting attractors, but also the structure and shape of attracting basin. There are gaps and holes appear in the boundary and inner of the basin of attraction. With the help of critical curves, it can be further analyzed that the sudden changes of these qualitative properties may be related to the fact that one or both ends of the critical curves cross the border of the basin of attraction.

Finally, through analysis on profit bifurcation diagrams of two manufacturers, we can know 
that with constant increase of the parameters, the system will become more disorderly and unpredictable. A conclusion will be obtained that the greater the values of product differentiation degree and service spillover effect are not the better. The two manufacturers should keep the value of these two factors in a relatively small range, which is conducive to steady operation of two manufacturers in long run.

\section{Declaration of competing interest}

The authors declare that the research was conducted in the absence of any conflict of interest.

\section{Acknowledgements}

Thanks for the great support of Research Center of Game theory and Economic Mathematics, Lanzhou Jiaotong University. The paper is supported by the Foundation of A Hundred Youth Talents Training Program of Lanzhou Jiaotong University, the Foundation of Humanities and Social Sciences from the Ministry of Education of China (No. 15YJC820007), and Science and technology project of Gansu Province (No.18ZC1LA008).

\section{Appendix A}

Proof (proof for Proposition 2). The Jacobian matrix $J\left(P_{1}\right)$ corresponding to $P_{1}=(0,0)$ has the form as

$$
J\left(P_{1}\right)=\left(\begin{array}{cc}
1+k_{1} A & 0 \\
0 & 1+k_{2} B
\end{array}\right)
$$

It is quite obvious that the matrix $J\left(P_{1}\right)$ is a diagonal matrix, the corresponding eigenvalues are $\lambda_{1}=1+k_{1} A$ and $\lambda_{2}=1+k_{2} B$. From the non-negativity of the adjustment speeds and auxiliary variables, we can know that $\left|\lambda_{1}\right|>1$ and $\left|\lambda_{2}\right|>1$. Therefore, the point $P_{1}=(0,0)$ is an unstable node.

Proof (proof for Proposition 3). The corresponding Jacobian matrix $J\left(P_{2}\right)$ of the boundary equilibrium point $P_{2}=\left(0, \frac{B}{2 b}\right)$ can be expressed as

$$
J\left(P_{2}\right)=\left(\begin{array}{cc}
1+k_{1}\left(A+\frac{\gamma B}{2 b}\right) & 0 \\
\frac{k_{2} \gamma B}{2 b} & 1-k_{2} B
\end{array}\right) .
$$

The matrix $J\left(P_{2}\right)$ is a lower triangular matrix, and the corresponding two eigenvalues can be obtained directly as $\lambda_{1}=1+k_{1}\left(A+\frac{\gamma B}{2 b}\right)$ and $\lambda_{2}=1-k_{2} B$, respectively. From the non-negativity 
of the parameters, we can see that $\left|\lambda_{1}\right|>1$, while the relation between the moduli of $\lambda_{2}$ and the constant 1 needs to discuss a step further. When the adjustment speed $k_{2}$ satisfies the condition that $k_{2}<\frac{2}{B}$, we can easily get that $\left|\lambda_{2}\right|<1$, then the point $P_{2}=\left(0, \frac{B}{2 b}\right)$ is a saddle point. Otherwise, $\left|\lambda_{2}\right|>1$ and $P_{2}=\left(0, \frac{B}{2 b}\right)$ is an unstable node.

Because of the symmetry of the structure of the two boundary equilibrium points $P_{2}=\left(0, \frac{B}{2 b}\right)$ and $P_{3}=\left(\frac{A}{2 b}, 0\right)$, they have similar stable properties. Hence, we will not describe in detail the process of determining the local stability of $P_{3}=\left(\frac{A}{2 b}, 0\right)$ in detail, but only give the corresponding Jacobian matrix $J\left(P_{3}\right)$ is

$$
J\left(P_{3}\right)=\left(\begin{array}{cc}
1-k_{1} A & \frac{k_{1} \gamma A}{2 b} \\
0 & 1+k_{2}\left(B+\frac{\gamma A}{2 b}\right)
\end{array}\right) .
$$

\section{References}

[1]O. Shy, Industrial Organization: Theory and Application. MIT Press, Cambridge, MA (1995).

[2]L. Fanti, N. Meccheri, Profits and competition under alternative technologies in a unionized duopoly with product differentiation, Res. Econ. 68 (2014) 157-168.

[3]A. Caplin, B. Nalebuff, Aggregation and Imperfect Competition : On the Existence of Equilibrium, The Econometric Society. 59 (1991) 25-59.

[4]L. Liu, X.H. Wang, C. Zeng, Endogenous Horizontal Product Differentiation in a Mixed Duopoly, Rev. Ind. Organ. 56 (2020) 435-462.

[5]J. Zhang, Q. Da, Y. Wang, The dynamics of Bertrand model with bounded rationality, Chaos, Solitons and Fractals. 39 (2009) 2048-2055.

[6]H.S. Bierman, L. Fernandez, Game Theory with Economic Applications 2nd edition. Reading, MA: Addison-Wesley. (1998)

[7]E. Ahmed, A.A. Elsadany, T. Puu, On Bertrand duopoly game with differentiated goods, Appl. Math. Comput. 251 (2015) 169-179.

[8]M. Anufriev, D. Kopányi, J. Tuinstra, Learning cycles in Bertrand competition with differentiated commodities and competing learning rules, J. Econ. Dyn. Control. 37 (2013) 2562-2581.

[9]X. Zhang, D. Sun, S. Ma et al., The dynamics of a quantum Bertrand duopoly with differentiated products and heterogeneous expectations, Physica A (2020) Paper No.124878.

[10]L. Fanti, L. Gori, The dynamics of a differentiated duopoly with quantity competition, Econ. Model. 29 (2012) 421-427.

[11]A. Agliari, A.K. Naimzada, N. Pecora, Nonlinear dynamics of a Cournot duopoly game with differentiated products, Appl. Math. Comput. 281 (2016) 1-15. 
[12]B.C. Giri, B.R. Sarker, Coordinating a two-echelon supply chain with price and service level, Oper. Res. 16 (2015) 71-88.

[13]J. Shin, How Does Free Riding on Customer Service Affect Competition ?, Marketing Science. 26 (2007) 488-503.

[14]D.Z Wu, Implications of Reduced Search Cost and Free Riding in E-Commerce, Marketing Science. 23 (2004) 255-262.

[15]L.G. Telser, WHY Should Manufactures Want Fair Trade?, JOURNAL OF LAW and ECONOMICS. 3 (1960) 86-105.

[16]F. Bernstein, J. Song, X. Zheng, Free Riding in a Multi-Channel Supply Chain, Naval Research Logistics. 56 (2006) 745-765.

[17]H. Tu, X. Mao, X. Wang, Complexity of a dynamic hybrid supply chain game model with a service factor, Nonlinear Dyn. 97 (2019) 2055-2066.

[18]Y. Yan, R. Zhao, Z. Liu, Strategic introduction of the marketplace channel under spillovers from online to offline sales, Eur. J. Oper. Res. 267 (2018) 65-77.

[19]H. Xiao, Competition in multichannel expert services in duopoly, J. Model. Manag. 14 (2019) 1088-1104.

[20]B. Xin, Y. Wang, Stability and Hopf Bifurcation of a Stochastic Cournot Duopoly Game in a Blockchain Cloud Services Market Driven by Brownian Motion, IEEE Access. 8 (2020) 4143241438.

[21]Y. Ding, X. Gao, C. Huang, D. Yang, Service Competition in an Online Duopoly Market, Omega. 77 (2017) 58-72.

[22]L. Fanti, L. Gori, C. Mammana, E. Michetti, The dynamics of a Bertrand duopoly with differentiated products: Synchronization, intermittency and global dynamics, Chaos, Solitons \& Fractals. 52 (2013) 73-86.

[23]L. Fanti, L. Gori, C. Mammana, E. Michetti, Multistability and complex basins in a nonlinear duopoly with price competition and relative profit delegation, Chaos. 26 (2016) 1-17.

[24]L. Fanti, L. Gori, M. Sodini, Nonlinear dynamics in a Cournot duopoly with relative profit delegation, Chaos, Solitons \& Fractals. 45 (2012) 1469-1478.

[25]G.I. Bischi, L. Stefanini, L. Gardini, Synchronization, intermittency and critical curves in a duopoly game, Math. Comput. Simul. 44 (1998) 559-585.

[26]G.I. Bischi, C. Mammana, L. Gardini, Multistability and cyclic attractors in duopoly games, Chaos, Solitons \& Fractals. 11 (2000) 543-564.

[27]G. Bischi, M. Kopel, Multistability and path dependence in a dynamic brand competition model, Chaos, Solitons \& Fractals. 18 (2003) 561-576.

[28]G.I. Bischi, F. Lamantia, Coexisting attractors and complex basins in discrete-time economic models, Nonlinear Dynamical Systems in Economics. 476 (2005) 187-231.

[29]Y. Zhang, W. Zhou, T. Chu, Y. Chu, J. Yu, Complex dynamics analysis for a two-stage Cournot duopoly game of semi-collusion in production, Nonlinear Dyn. 91 (2018) 819-835.

[30]J. Zhou, W. Zhou, T. Chu, Y. xiang Chang, M. jia Huang, Bifurcation, intermittent chaos and multi-stability in a two-stage Cournot game with R\&D spillover and product differentiation, Appl. Math. Comput. 341 (2019) 358-378.

[31] W. Zhou, N. Zhao, T. Chu, Y. Chang, Stability and Multistability of a Bounded Rational Mixed Duopoly Model with Homogeneous Product, Complexity. 2020 (2020) 1-16.

[32] C. Liu, R.-C. He, W. Zhou et al., Dynamic analysis of airline bidding game based on nonlinear 
cost, Physica A (2020), Paper No.125547.

[33] W. Zhou, J. Zhou, T. Chu, H. Li, A Dynamic Duopoly Cournot Model with R\&D Efforts and Its Dynamic Behavior Analysis, Complexity. 2020 (2020) 1-19.

[34]W. Zhou, X.X. Wang, On the Stability and Multistability in a Duopoly Game with R\&D Spillover and Price Competition, Discret. Dyn. Nat. Soc. 2019 (2019) 1-20.

[35]F. Cavalli, A. Naimzada, F. Tramontana, Nonlinear dynamics and global analysis of a heterogeneous Cournot duopoly with a local monopolistic approach versus a gradient rule with endogenous reactivity, Commun Nonlinear Sci Numer Simulat. 23 (2015) 245-262.

[36]Y. Cao, W. Zhou, T. Chu, Y. Chang, Global Dynamics and Synchronization in a Duopoly Game with Bounded Rationality and Consumer Surplus, Int. J. Bifurc. Chaos. 29 (2019) 1-22.

[37]X. Rao, Y. Chu, Y. Chang, Fractal structures in centrifugal flywheel governor system, Commun Nonlinear Sci Numer Simul. 50 (2017) 330-339.

[38]C. Manchein, R.M. da Silva, M.W. Beims, Proliferation of stability in phase and parameter spaces of nonlinear systems, Chaos. 27 (2017) Paper No.081101.

[39]Y. Pomear, P. Manneville, Intermittent transition to turbulence in dissipative dynamical systems, Commun. Math. Phys. 74 (1980) 189-197.

[40]C. Grebogi, E. Ott, F. Romeiras, Critical exponents for crisis-induced intermittency, Phys. Review A. 36 (1987) 5365-5380.

[41]C. Grebogi, E. Ott, Crises sudden changes in chaotic attractors and transient chaos, Phys. D. 7 (1983) 181-200. 
Figures

(a)
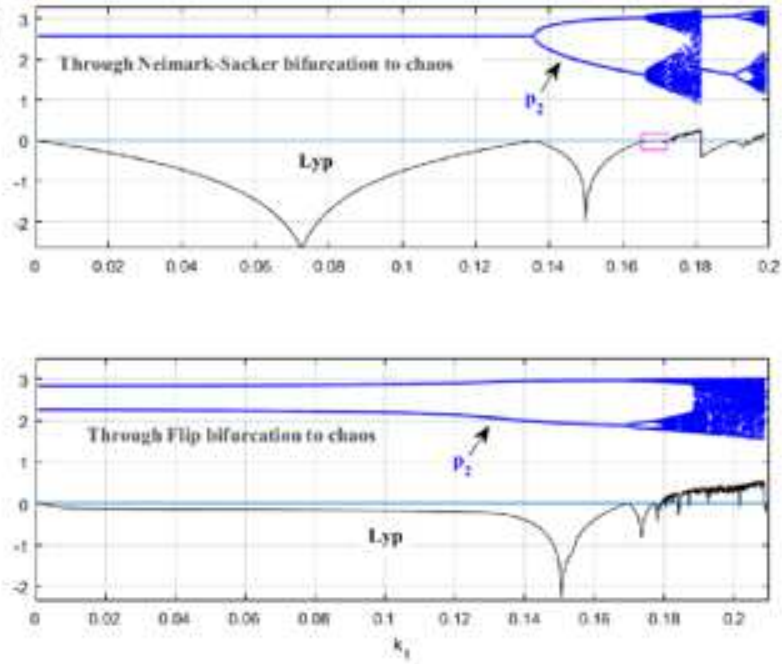

(c)

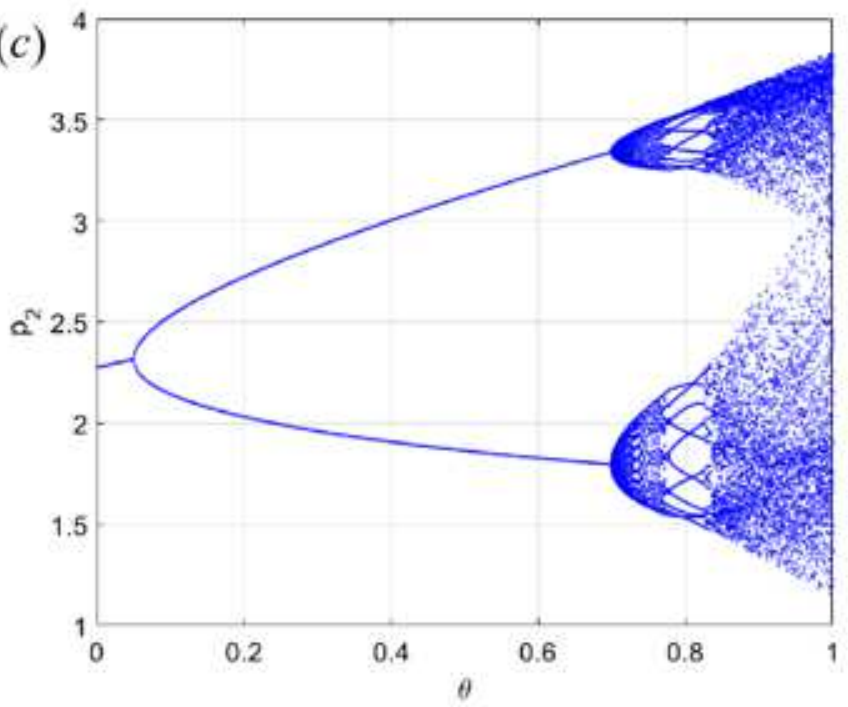

(b)
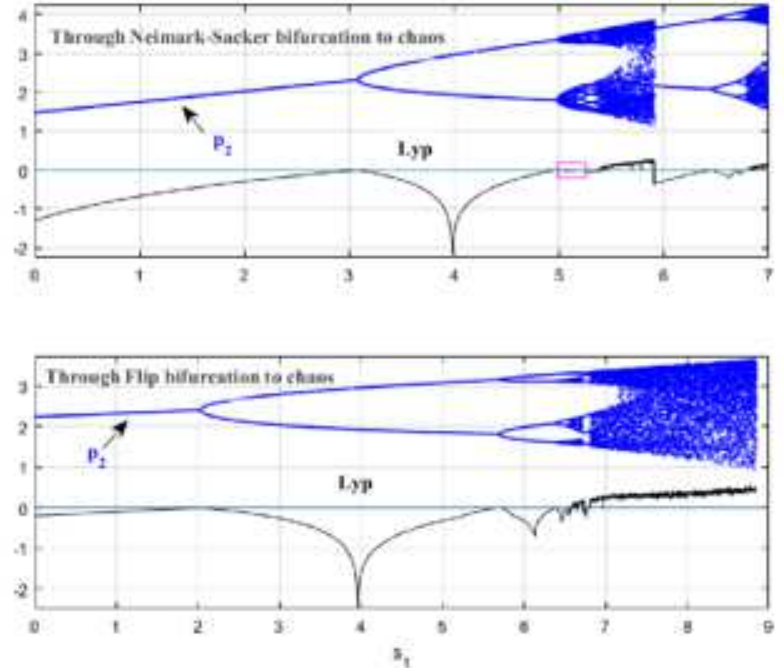

$(d)$

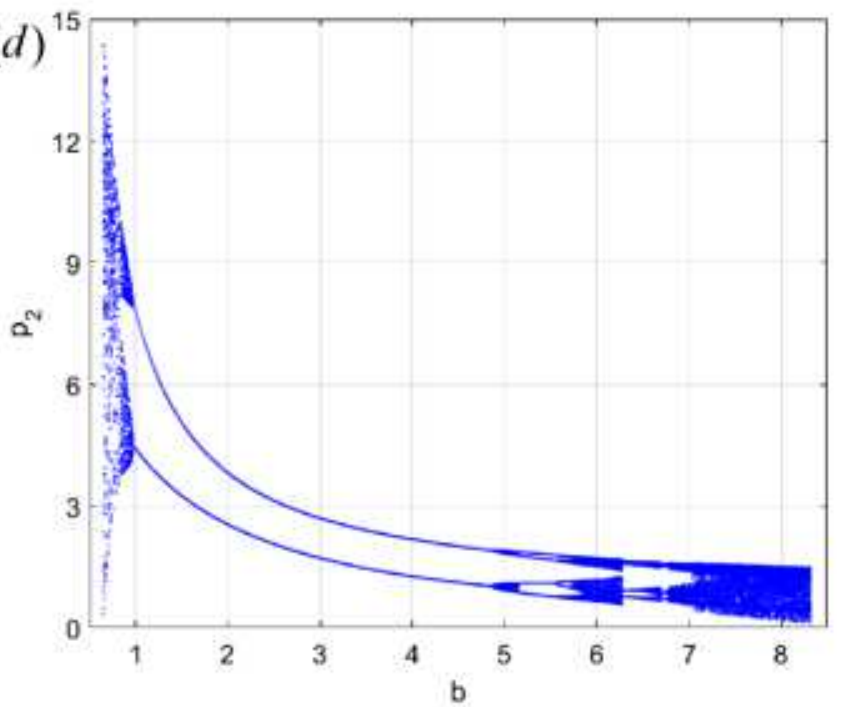

Figure 1

Please See the Manuscript Pdf File for the complete figure caption. 

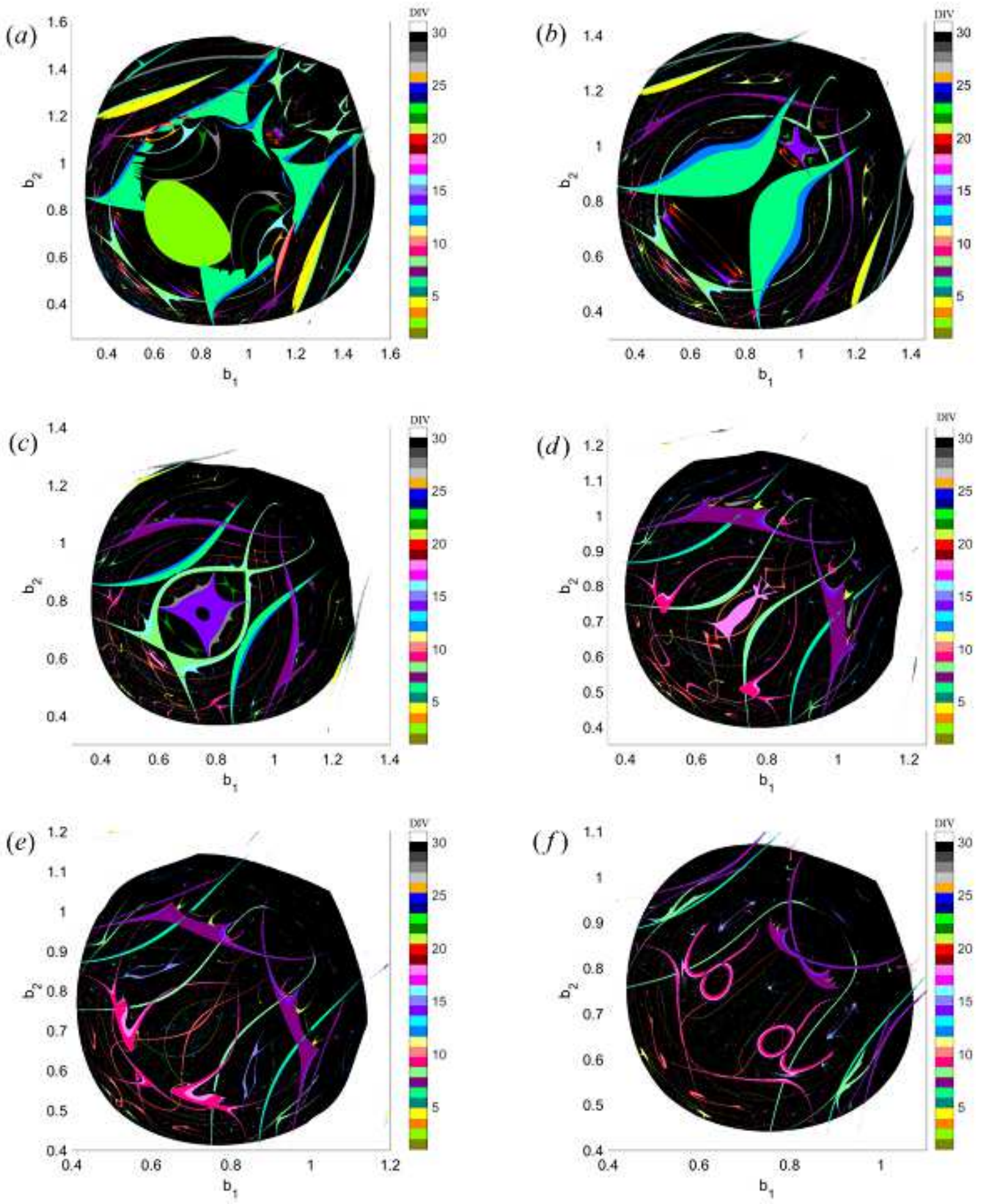

Figure 2

Please See the Manuscript Pdf File for the complete figure caption. 
(a)

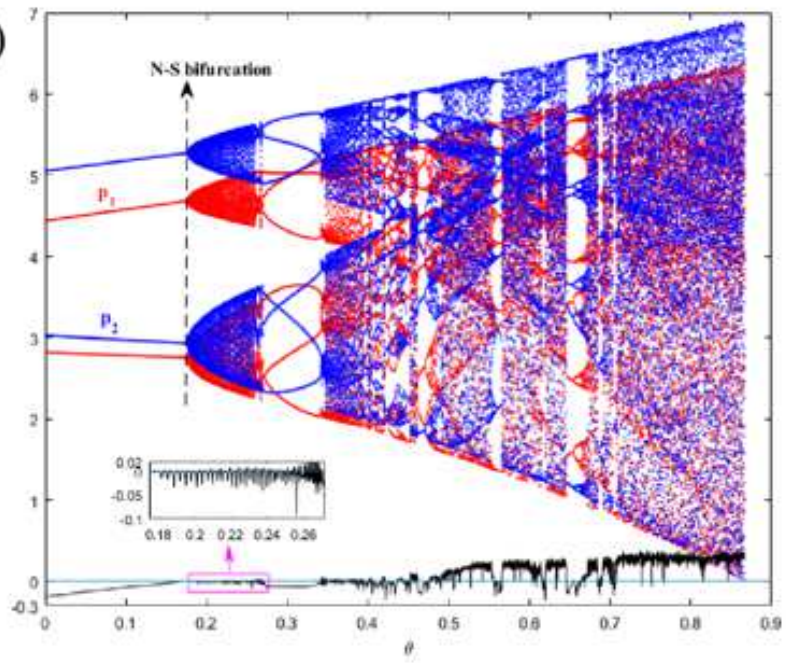

(b)

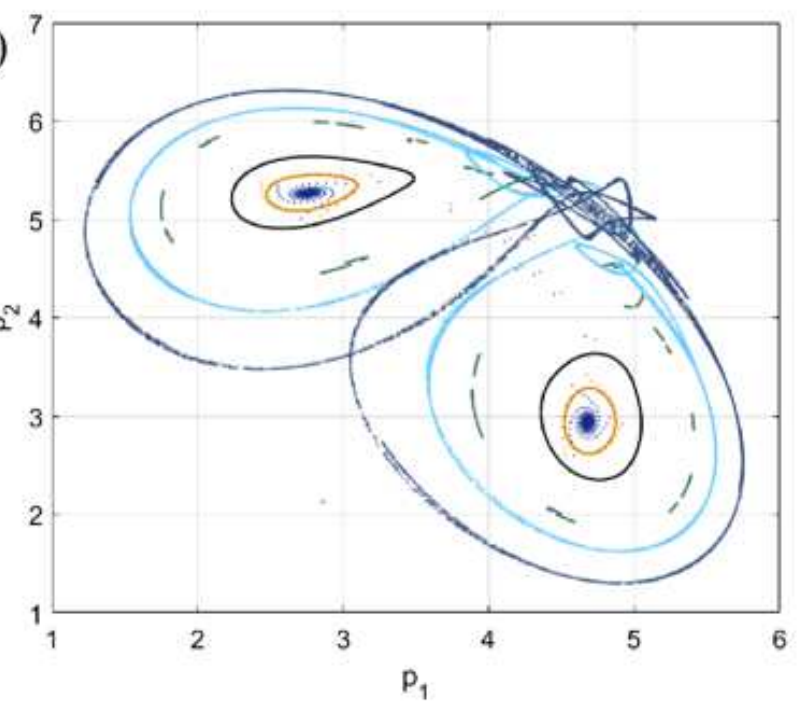

Figure 3

Please See the Manuscript Pdf File for the complete figure caption.
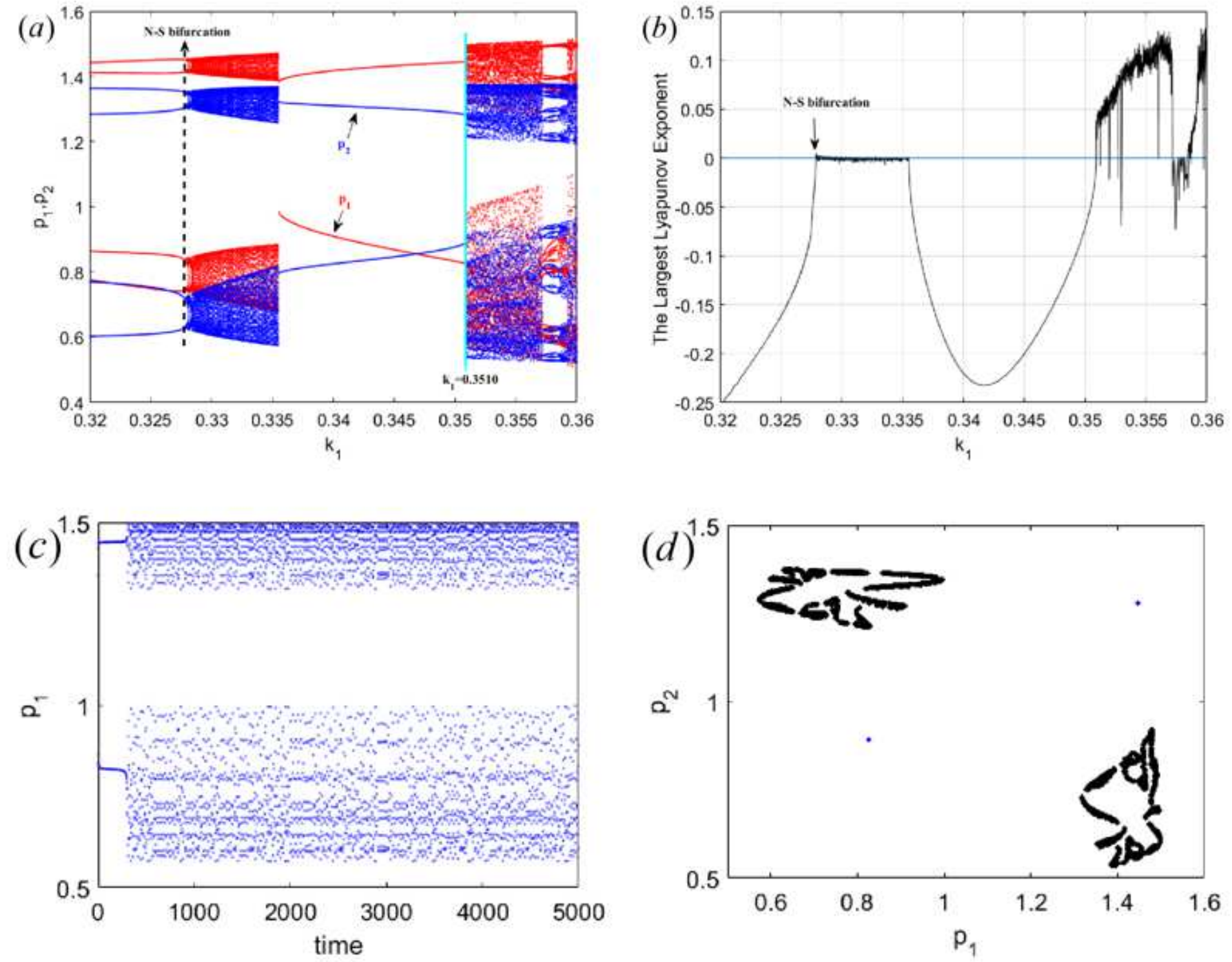
Figure 4

Please See the Manuscript Pdf File for the complete figure caption.
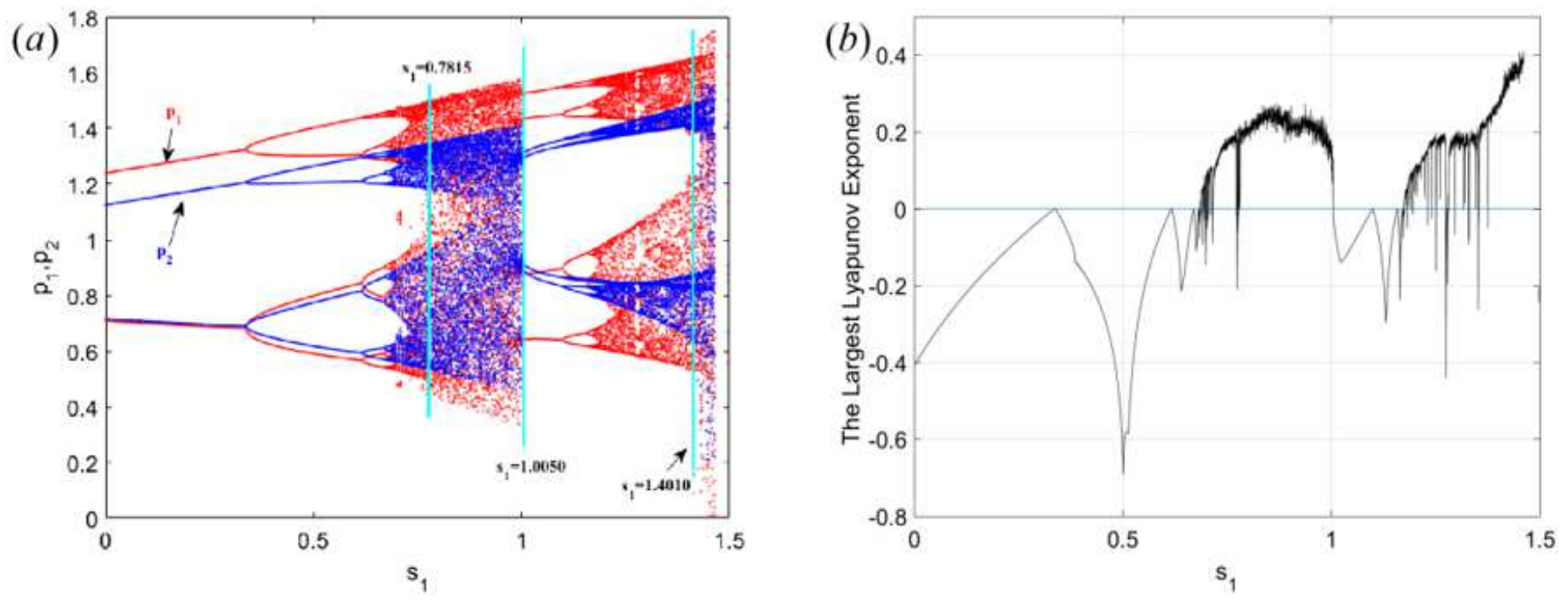

Figure 5

Please See the Manuscript Pdf File for the complete figure caption. 

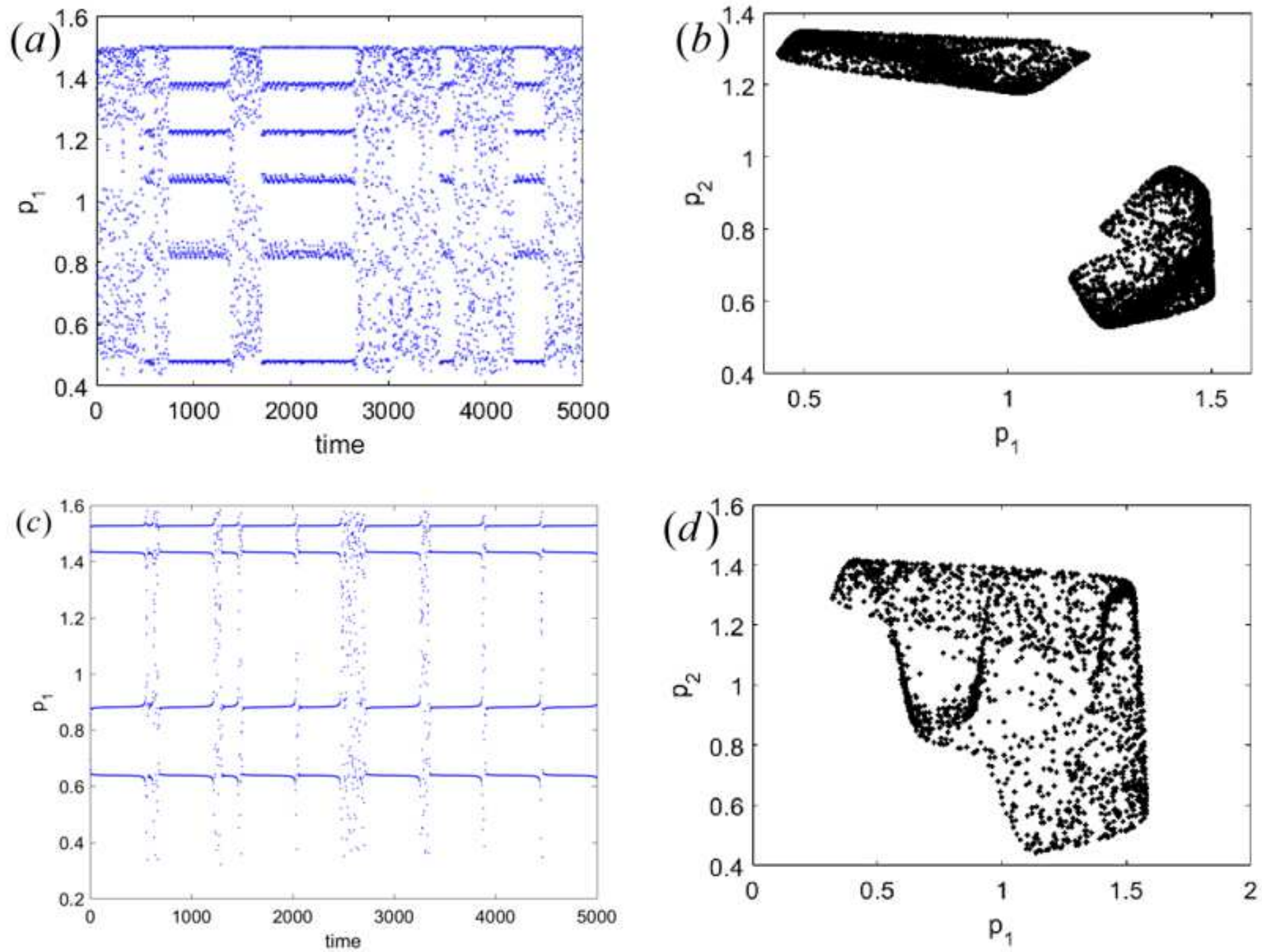

Figure 6

Please See the Manuscript Pdf File for the complete figure caption. 

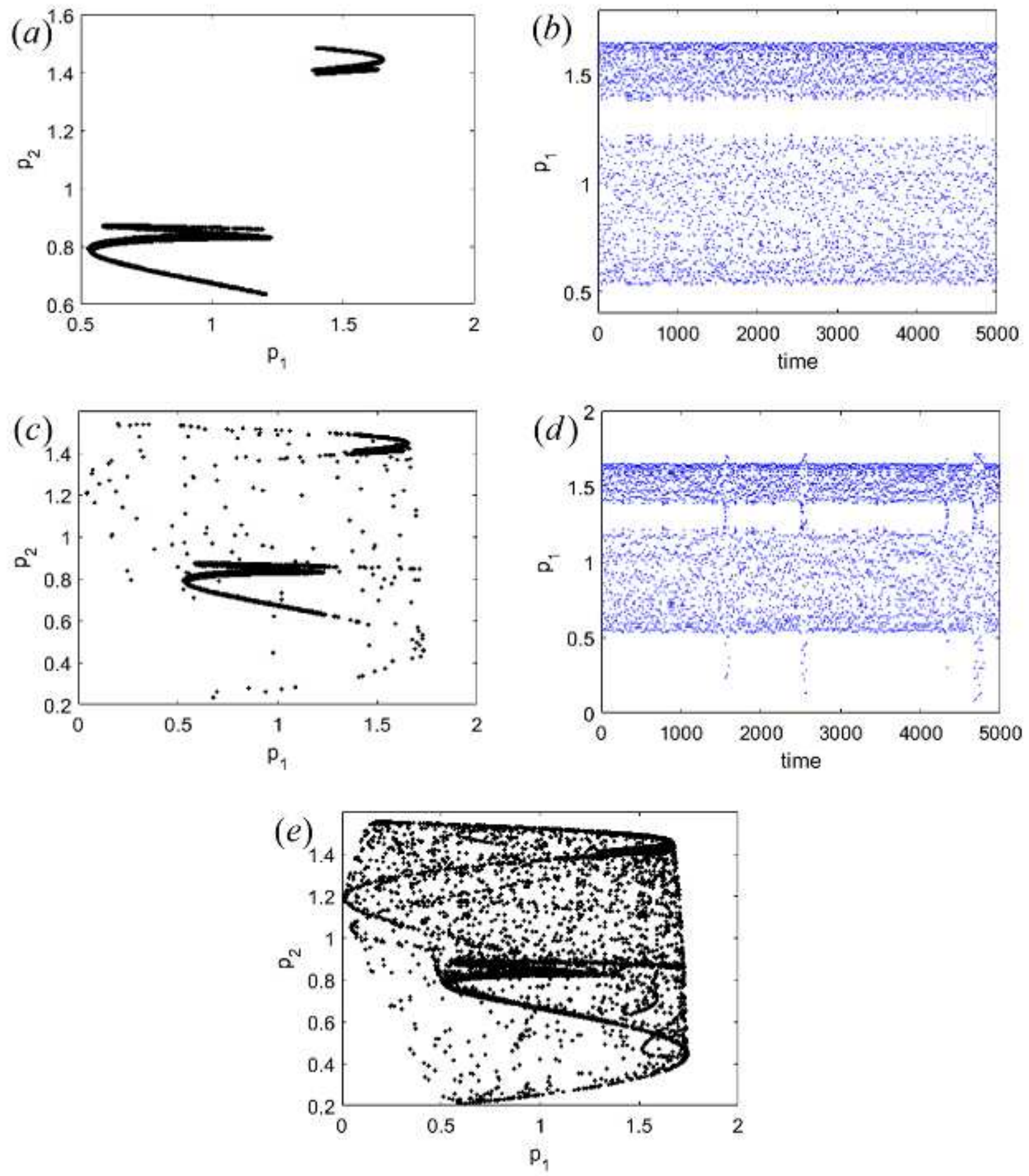

Figure 7

Please See the Manuscript Pdf File for the complete figure caption. 

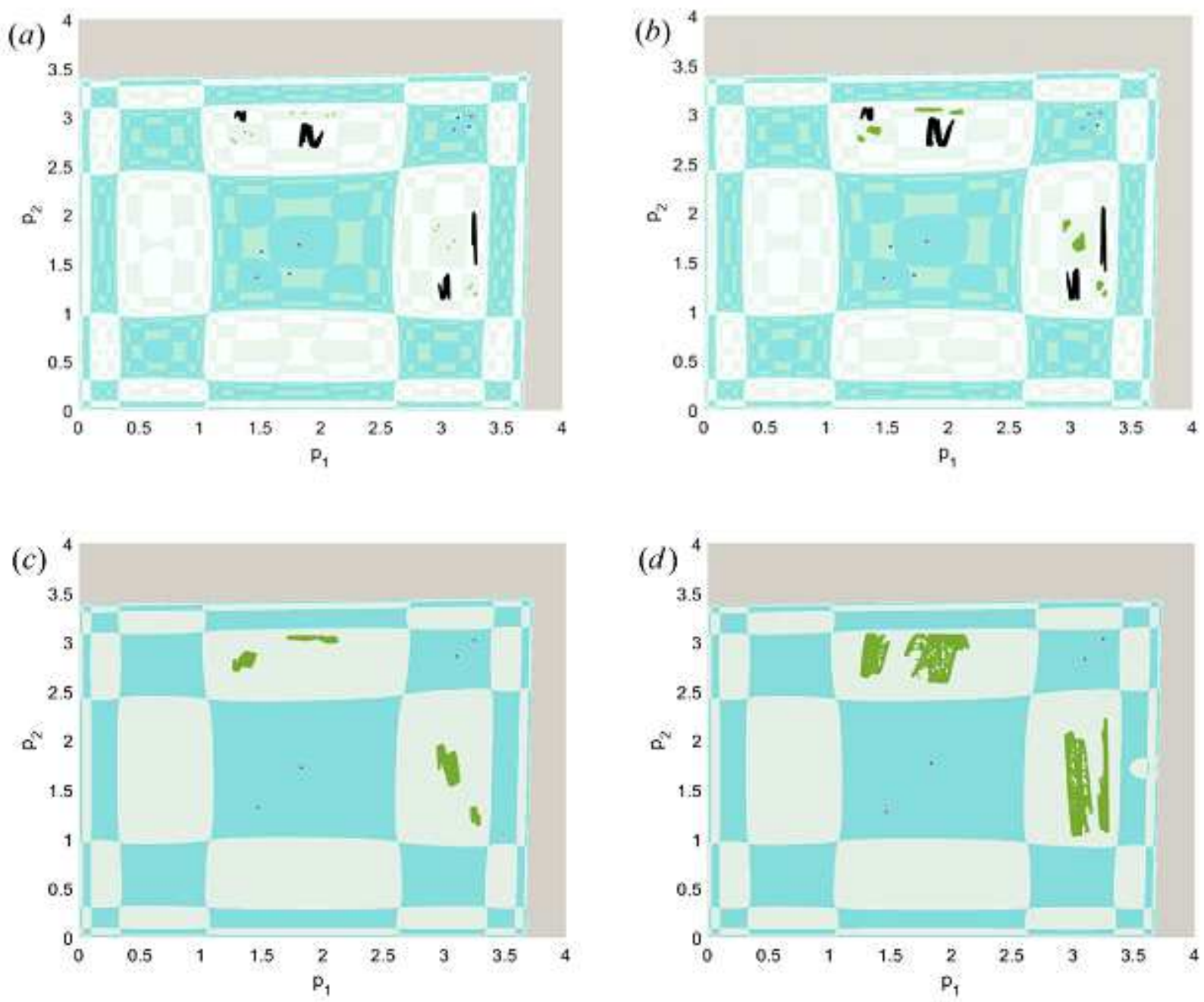

\section{Figure 8}

Please See the Manuscript Pdf File for the complete figure caption.
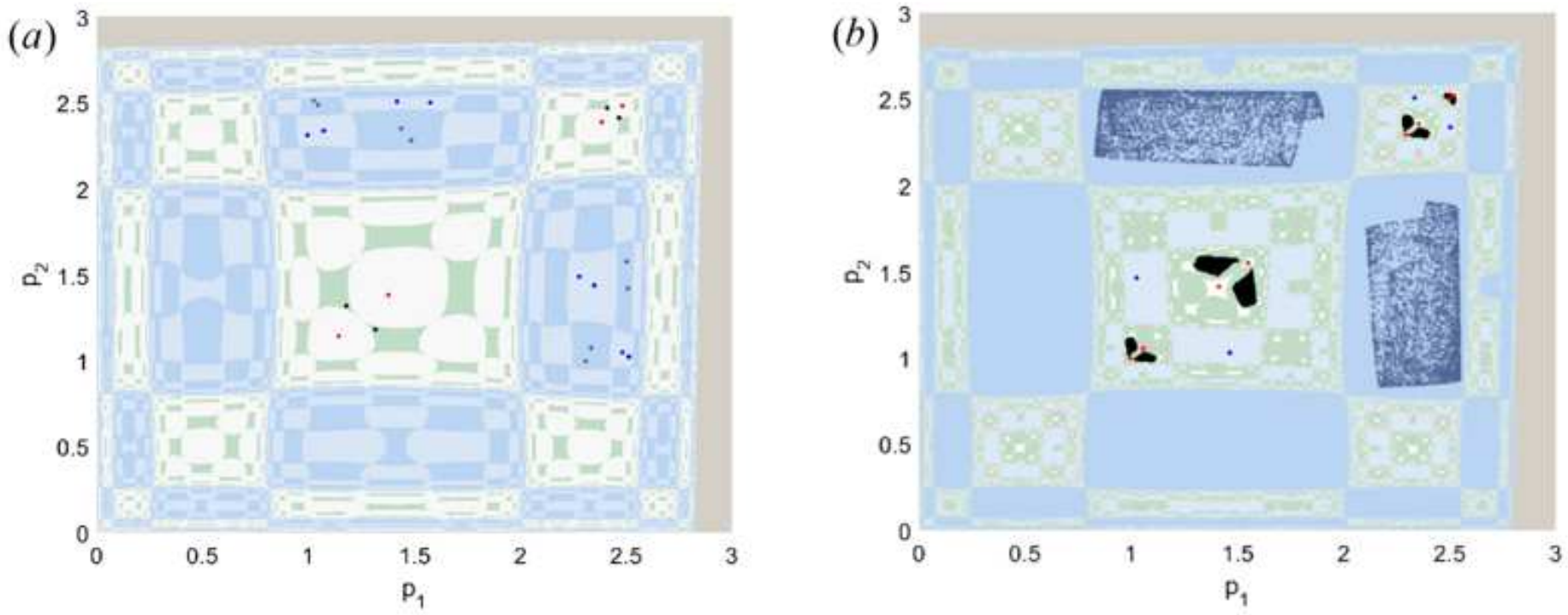

Figure 9 
Please See the Manuscript Pdf File for the complete figure caption.
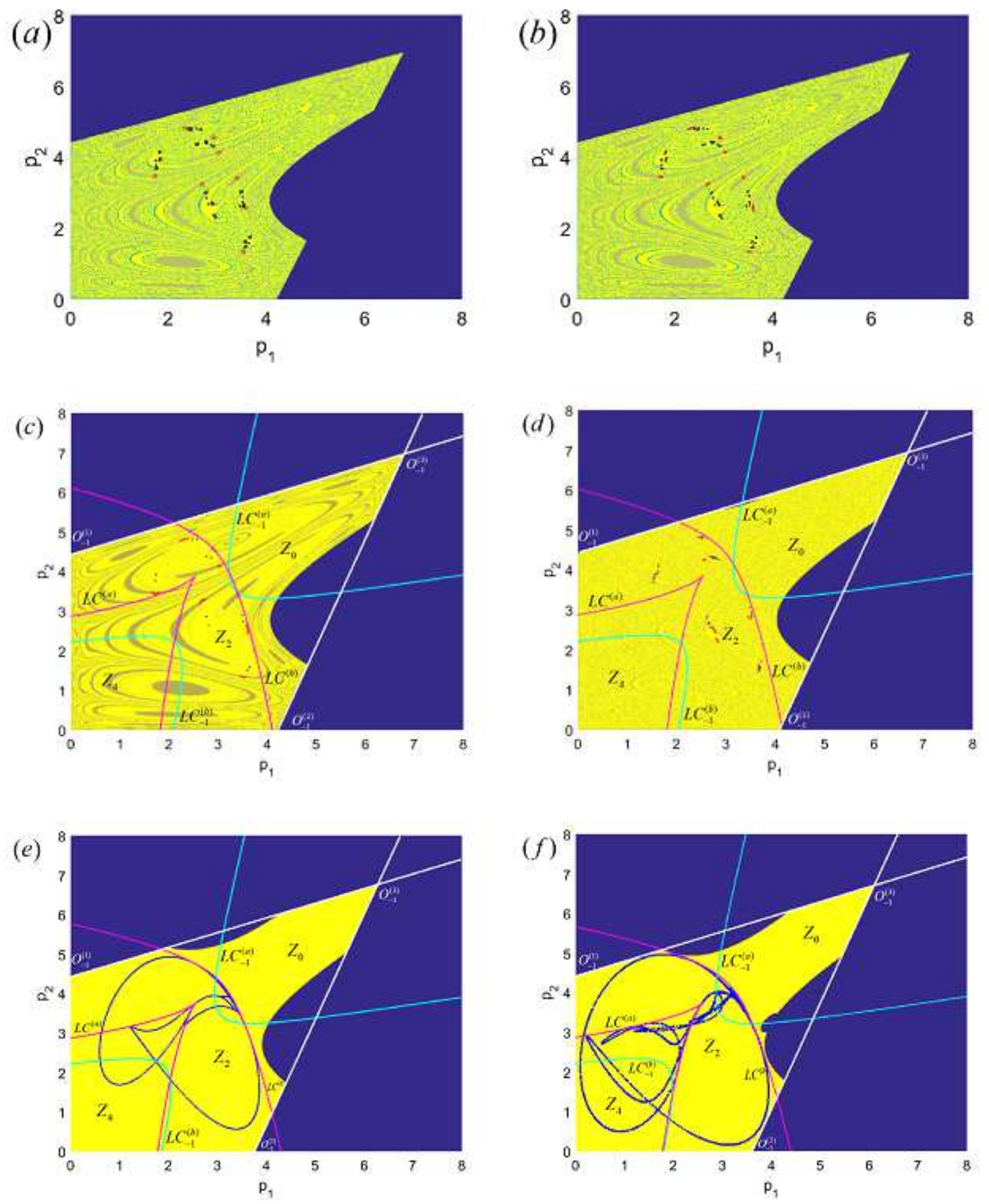

Figure 10

Please See the Manuscript Pdf File for the complete figure caption. 

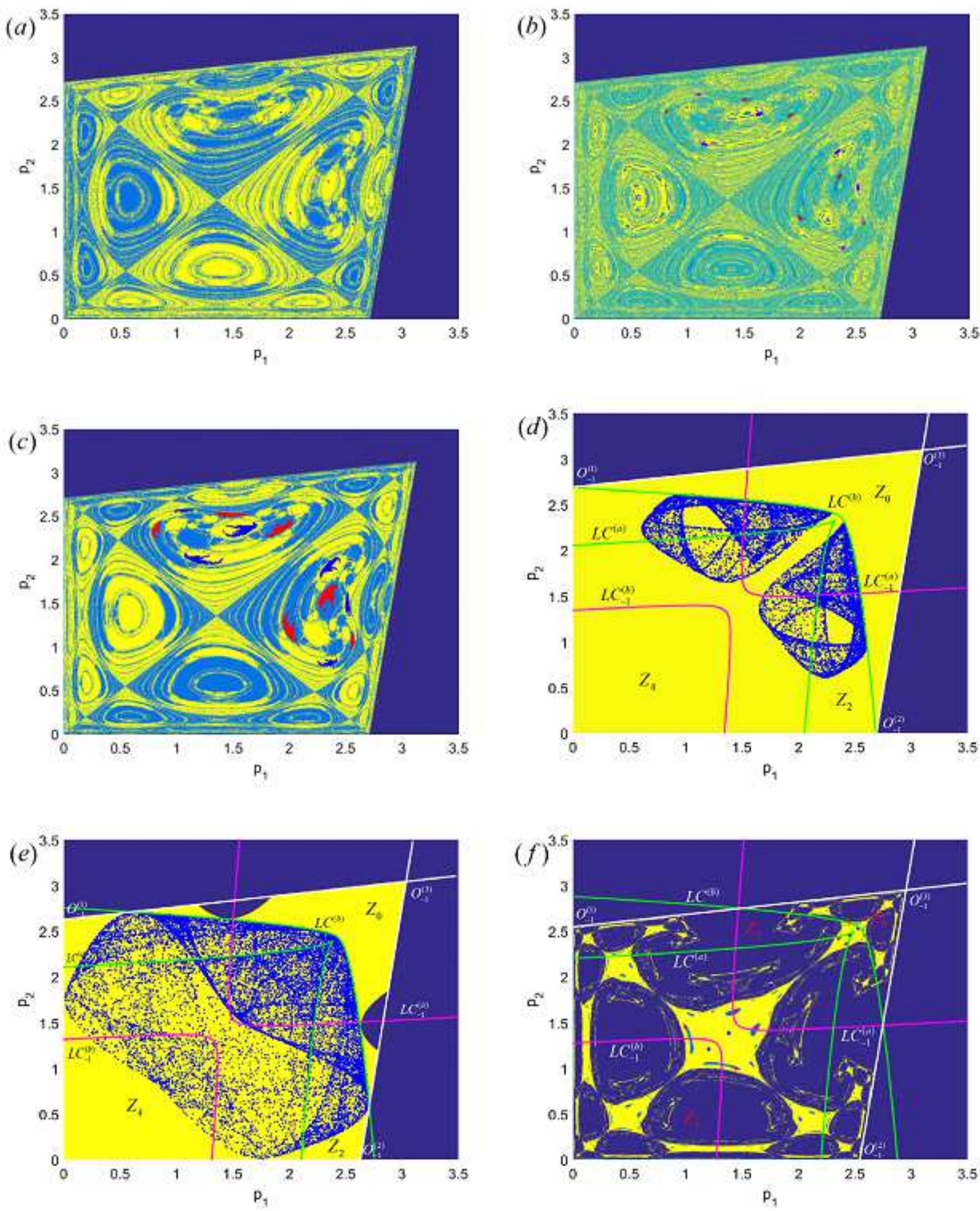

\section{Figure 11}

Please See the Manuscript Pdf File for the complete figure caption. 
(a)
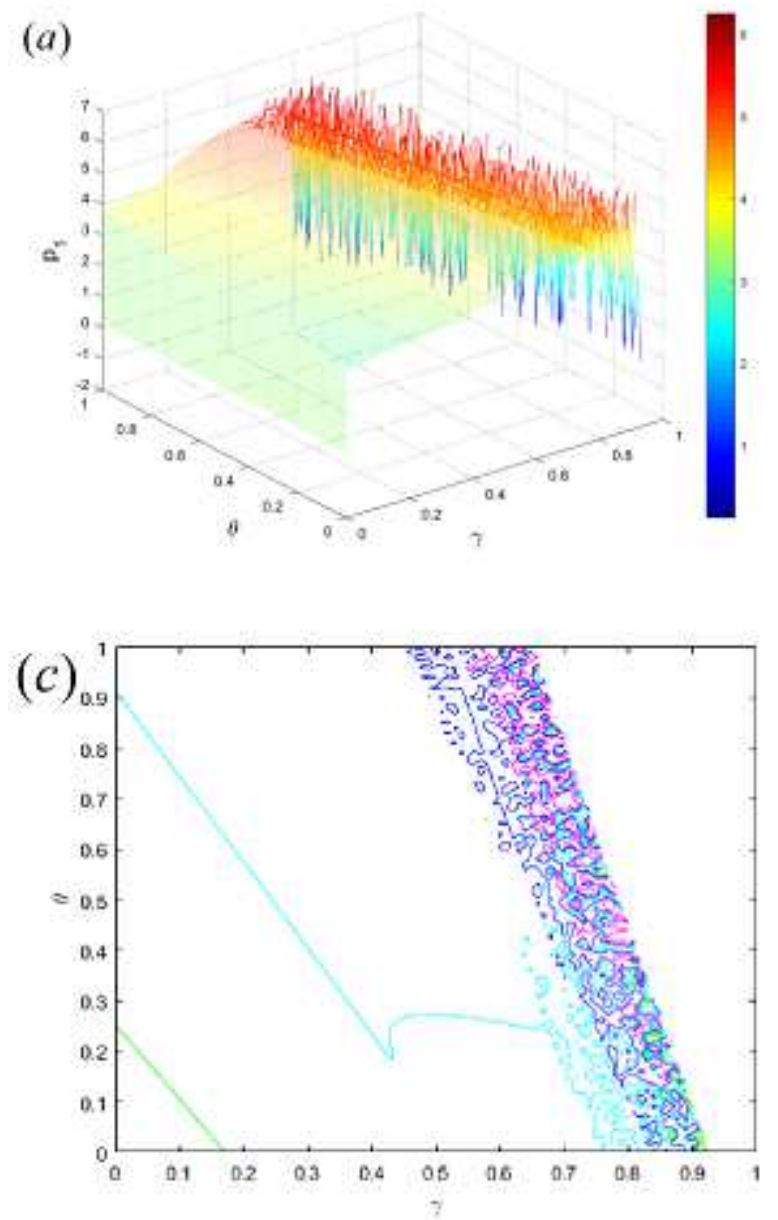

(e)

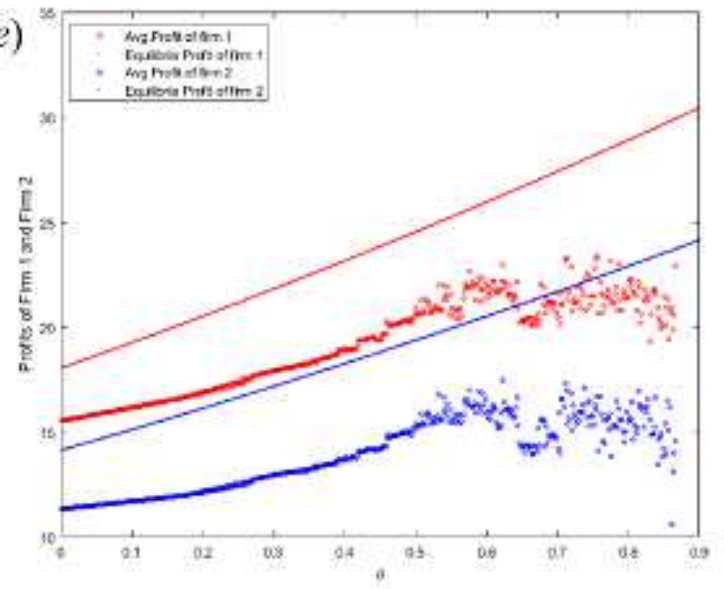

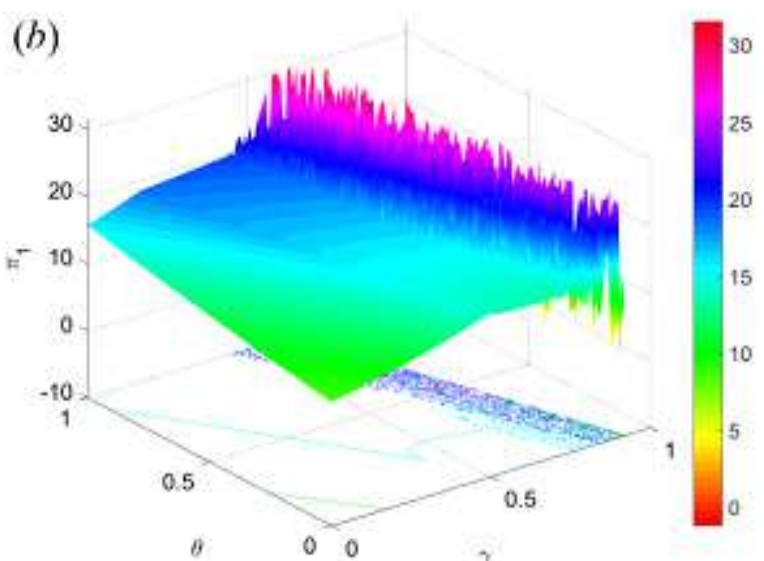
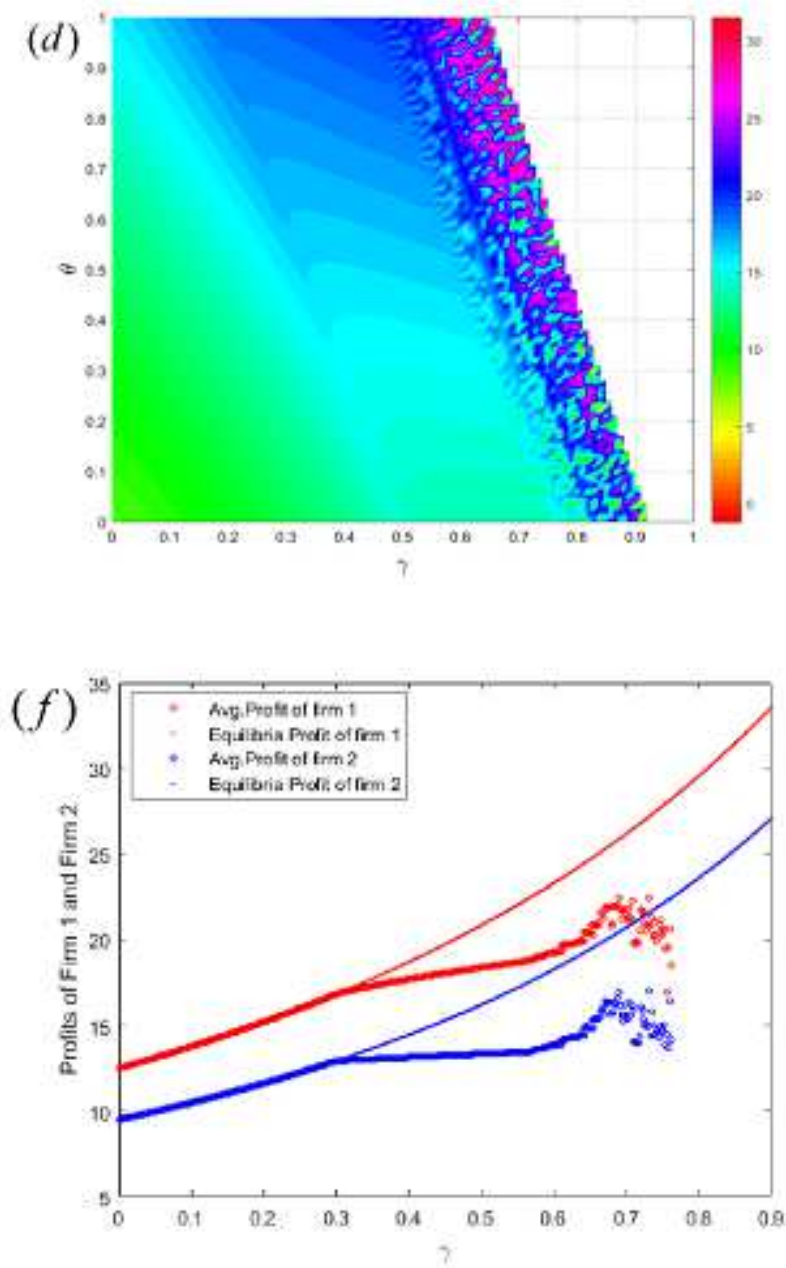

Figure 12

Please See the Manuscript Pdf File for the complete figure caption.

\section{Supplementary Files}

This is a list of supplementary files associated with this preprint. Click to download. 
- AppendixA.docx 